\title{
Cyanation of Phenol Derivatives with Aminoacetonitriles by Nickel Catalysis
}

\author{
Ryosuke Takise ${ }^{[a]}$ Kenichiro Itami, ${ }^{* a, b]}$ and Junichiro Yamaguchi ${ }^{[c]}$ \\ [a] Institute of Transformative Bio-Molecules (WPI-ITbM) and Graduate School of Science, Nagoya University, \\ Chikusa, Nagoya 464-8602, Japan. \\ [b] JST-ERATO, Itami Molecular Nanocarbon Project, Nagoya University, Chikusa, Nagoya 464-8602, Japan \\ [c] Department of Applied Chemistry, Waseda University, 3-4-1 Ohkubo, Shinjuku, Tokyo 169-8555, Japan \\ E-mail: junyamaguchi@waseda.jp, itami@chem.nagoya-u.ac.jp
}

\section{Table of Contents}

1. General

S2

2. Synthesis of Starting Materials

S3-S7

3. Ni-Catalyzed Cyanation of Aryl Carbamates with Aminoacetonitrile 2 S8-S11

4. Ni-Catalyzed Cyanation of Aryl Pivalates with Aminoacetonitrile 2 S11-S16

5. Ni-Catalyzed Cyanation of Vinyl Carbamate with Aminoacetonitrile 2 S16-S17

6. Sequential Cross-coupling for the Synthesis of $\mathbf{5 C B}$

S17-S18

7. Ligand screening

S19

9. $\quad{ }^{1} \mathrm{H}$ and ${ }^{13} \mathrm{C}$ Spectra

S20-S108 


\section{General}

Unless otherwise noted, all reactants or reagents including dry solvents were obtained from commercial suppliers and used as received. $\mathrm{NiBr}_{2}$ was obtained from Strem Chemicals and $\mathrm{K}_{3} \mathrm{PO}_{4}$ and Zn powder was obtained from Wako Chemicals. Unless otherwise noted, all reactions were performed with dry solvents under an atmosphere of argon in dried glassware using standard vacuum-line techniques. All cyanation reactions were performed in $20-\mathrm{mL}$ glass vessel tubes equipped with $\mathrm{J}$. Young ${ }^{\circledR}$ O-ring tap and heated in an 8-well reaction block (heater + magnetic stirrer) unless otherwise noted. All work-up and purification procedures were carried out with reagent-grade solvents in air.

Analytical thin-layer chromatography (TLC) was performed using E. Merck silica gel $60 \mathrm{~F}_{254}$ precoated plates $(0.25 \mathrm{~mm})$. The developed chromatogram was analyzed by UV lamp $(254 \mathrm{~nm})$. Flash column chromatography was performed with E. Merck silica gel 60 (230-400 mesh). Preparative thin-layer chromatography (PTLC) was performed using Wakogel B5-F silica coated plates $(0.75 \mathrm{~mm})$ prepared in our laboratory. Preparative gel permeation chromatography (GPC) was performed with a JAI LC-9204 instrument equipped with JAIGEL-1H/JAIGEL-2H columns using chloroform as an eluent. Gas chromatography (GC) analysis was conducted on a Shimadzu GC-2010 instrument equipped with a HP-5 column (30 m $\times 0.25 \mathrm{~mm}$, Hewlett-Packard) with dodecane as an internal standard. GCMS analysis was conducted on a Shimadzu GCMS-QP2010 instrument equipped with a Restec-5HT column (30 $\mathrm{m} \times 0.25 \mathrm{~mm}$, Hewlett-Packard). The high-resolution mass spectra (HRMS) were conducted on Thermo Fisher Scientific Exactive. Infrared spectra were recorded on a JASCO FTIR-6100 spectrometer. Nuclear magnetic resonance (NMR) spectra were recorded on a JEOL JNM-ECA-600 ( $\left.{ }^{1} \mathrm{H} 600 \mathrm{MHz},{ }^{13} \mathrm{C} 150 \mathrm{MHz},{ }^{31} \mathrm{P} 243 \mathrm{MHz}\right)$ spectrometer and a JEOL JNM-ECA-400

$\left({ }^{1} \mathrm{H} 400 \mathrm{MHz},{ }^{13} \mathrm{C} 100 \mathrm{MHz},{ }^{31} \mathrm{P} 162 \mathrm{MHz}\right)$ spectrometer. Chemical shifts for ${ }^{1} \mathrm{H}$ NMR are expressed in parts per million $(\mathrm{ppm})$ relative to tetramethylsilane $(\delta 0.00 \mathrm{ppm})$ or residual peak of DMSO- $d_{6}(\delta$ $2.50 \mathrm{ppm})$. Chemical shifts for ${ }^{13} \mathrm{C}$ NMR are expressed in ppm relative to $\mathrm{CDCl}_{3}(\delta 77.0 \mathrm{ppm})$ or DMSO- $d_{6}(\delta 39.5 \mathrm{ppm})$. Data are reported as follows: chemical shift, multiplicity $(\mathrm{s}=$ singlet, $\mathrm{d}=$ doublet, $\mathrm{dd}=$ doublet of doublets, $\mathrm{ddd}=$ doublet of doublets of doublets, $\mathrm{t}=$ triplet, $\mathrm{dt}=$ doublet of triplets, $\mathrm{td}=$ triplet of doublets, $\mathrm{q}=$ quartet, $\mathrm{p}=$ quintet, $\mathrm{se}=$ sextet $\mathrm{m}=$ multiplet, $\mathrm{bs}=$ broad signal), coupling constant $(\mathrm{Hz})$, and integration. 


\section{Synthesis of Starting Materials}

Note: $\mathbf{1} \mathrm{A}^{[1]}, \mathbf{1 C}^{[1]}, \mathbf{1 D}^{[1]}, \mathbf{1 G}^{[1]}, \mathbf{1 I}^{[1]}, \mathbf{1 J}^{[1]}, \mathbf{1} \mathrm{K}^{[2]}, \mathbf{1 L}^{[3]}, \mathbf{1 P}^{[4]}, \mathbf{1 Q}^{[3]}, \mathbf{1} \mathbf{T}^{[4]}, \mathbf{1 U}^{[3]}, \mathbf{1} \mathbf{W}^{[4]}, \mathbf{1} \mathbf{Y}^{[5]}, \mathbf{1 A} \mathbf{A}^{[1]}$, $\mathbf{1} \mathbf{A B}{ }^{[6]}, \mathbf{1} \mathbf{A D}^{[6]}$ were synthesized according to procedures reported in the literature.

\section{2-1. General Procedure for the Synthesis of Aryl Carbamates}

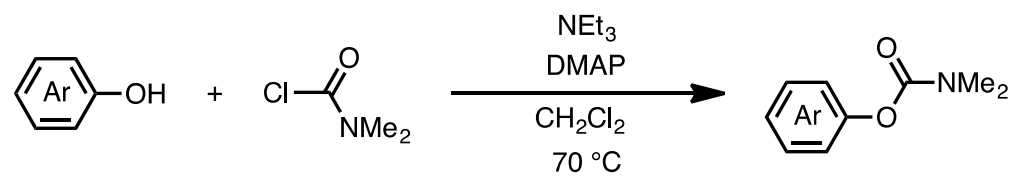

Method A: To a solution of the corresponding phenols and a piece of $N, N$-dimethyl-4-aminopyridine (DMAP) in dichloroethane $(0.50 \mathrm{M})$ were added $\mathrm{Et}_{3} \mathrm{~N}$ (1.2 equiv) and $N, N$-dimethylcarbamoyl chloride (1.2 equiv). This mixture was stirred overnight at $70{ }^{\circ} \mathrm{C}$. The reaction mixture was quenched by the addition of saturated $\mathrm{NaHCO}_{3} \mathrm{aq}$, and then the mixture was extracted three times with $\mathrm{CH}_{2} \mathrm{Cl}_{2}$. The combined organic layer was dried over $\mathrm{Na}_{2} \mathrm{SO}_{4}$, and concentrated in vacuo. The residue was purified by flash silica-gel column chromatography to afford the corresponding product.

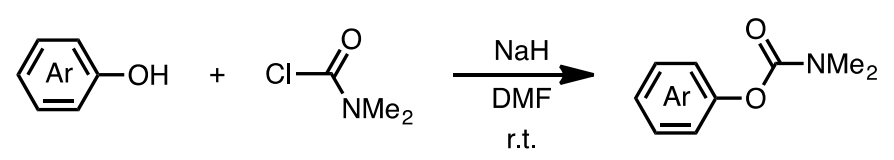

Method B: $\mathrm{NaH}$ (60\% dispersion in paraffin liquid: 1.3 equiv) was placed in a 100-mL 2-necked flask under a stream of $\mathrm{N}_{2}$, and DMF $(0.42 \mathrm{M})$ was added to the flask. To this suspension, the corresponding phenols (1.0 equiv) was added. The resulting mixture was stirred at room temperature for $5 \mathrm{~min}$. A solution of $N, N$-dimethylcarbamoyl chloride (1.0 equiv) was added, and then the mixture was stirred for an additional $30 \mathrm{~min}$. The reaction was quenched by the addition of water. The mixture was extracted three times with EtOAc, and the combined organic layer was washed with water and brine, dried over $\mathrm{Na}_{2} \mathrm{SO}_{4}$, and then filtered. The filtrate was concentrated in vacuo. The crude residue was purified by flash silica-gel column chromatography to afford the corresponding product.<smiles>CC(=O)Oc1cccc(-c2ccccc2)c1</smiles>

[1] K. Huang, D.-G. Yu, S.-F. Zheng, Z.-H. Wu, Z.-J. Shi, Chem. Eur. J. 2011, 17, 786.

[2] L. Wu, B.-J. Li, Z.-H. Wu, X.-Y. Lu, B.-T. Guan, B.-Q. Wang, K.-Q. Zhao, Z.-J. Shi, Org. Lett. 2010, 12, 884.

[3] C. Zarate, R. Martin, J. Am. Chem. Soc. 2014, 136, 2236.

[4] K. W. Quasdorf, X. Tian, N. K. Garg, J. Am. Chem. Soc. 2008, 130, 14422.

[5] R. Takise, K. Muto, J. Yamaguchi, K. Itami, Angew. Chem. Int. Ed. 2014, 53, 6791.

[6] K. Muto, T. Hatakeyama, J. Yamaguchi, K. Itami, Chem. Sci. 2015, 6, 6792. 


\section{[1,1'-Biphenyl]-3-yl dimethylcarbamate (1B)}

Method B: Purification by flash silica-gel column chromatography (hexane/EtOAc $=3: 1$ ) afforded $1 \mathbf{E}$ as a colorless liquid (1.18 g, $5.0 \mathrm{mmol}$ scale, $98 \%$ yield). ${ }^{1} \mathrm{H}$ NMR (400 MHz, $\left.\mathrm{CDCl}_{3}\right) \quad \delta$ 7.65-7.55 (m, 2H), 7.48-7.29 (m, 6H), 7.15-7.05 (m, 1H). $3.11(\mathrm{~s}, 3 \mathrm{H}), 3.03(\mathrm{~s}, 3 \mathrm{H}) ;{ }^{13} \mathrm{C}$ NMR $(100$ $\mathrm{MHz}, \mathrm{CDCl}_{3}$ ) $\delta \quad 154.9,151.9,142.6,140.3,129.5,128.7,127.5,127.2,123.9,120.5,36.7,36.4$ (one peak is overlapping); HRMS (ESI) $\mathrm{m} / z$ calcd for $\mathrm{C}_{15} \mathrm{H}_{16} \mathrm{NO}_{2}^{+}[\mathrm{M}+\mathrm{H}]^{+}: 242.1176$ found 242.1174.

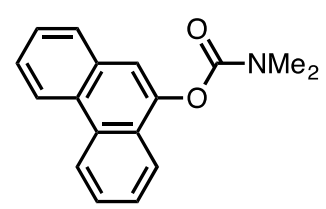

\section{Phenanthren-9-yl dimethylcarbamate (1E)}

Method B: Purification by flash silica-gel column chromatography (hexane/EtOAc $=5: 1$ ) afforded $1 \mathbf{E}$ as a white solid (362.7 mg, $2.8 \mathrm{mmol}$ scale, $49 \%$ yield). ${ }^{1} \mathrm{H}$ NMR (400 $\left.\mathrm{MHz}, \mathrm{CDCl}_{3}\right) \quad \delta$ $8.70(\mathrm{~d}, J=8.4 \mathrm{~Hz}, 1 \mathrm{H}), 8.65(\mathrm{~d}, J=8.4 \mathrm{~Hz}, 1 \mathrm{H}), 8.01(\mathrm{~d}, J=8.0 \mathrm{~Hz}, 1 \mathrm{H}), 7.84(\mathrm{~d}, J=7.6 \mathrm{~Hz}, 1 \mathrm{H})$, 7.71-7.55 (m, 5H), 3.31 (s, 3H), $3.10(\mathrm{~s}, 3 \mathrm{H}) ;{ }^{13} \mathrm{C} \mathrm{NMR}\left(100 \mathrm{MHz}, \mathrm{CDCl}_{3}\right) \quad \delta \quad 154.9,145.5,131.7$, 131.5, 128.7, 128.4, 127.2, 127.0, 126.9, 126.8, 126.1, 122.9, 122.6, 121.9, 117.6, 36.9, 36.6; HRMS (ESI) $m / z$ calcd for $\mathrm{C}_{17} \mathrm{H}_{16} \mathrm{NO}_{2}^{+}[\mathrm{M}+\mathrm{H}]^{+}: 266.1176$ found 266.1176 .

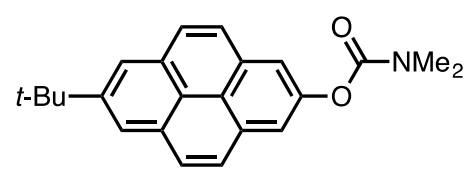

\section{7-(tert-Butyl)pyren-2-yl dimethylcarbamate (1F)}

Method A: Purification by flash silica-gel column chromatography (hexane/EtOAc $=10: 1 \sim 3: 1$ ) afforded $\mathbf{1 F}$ as a white solid (309.0 mg, $0.92 \mathrm{mmol}$ scale, $97 \%$ yield). ${ }^{1} \mathrm{H}$ NMR (400 $\left.\mathrm{MHz}, \mathrm{CDCl}_{3}\right) \quad \delta$ $8.22(\mathrm{~s}, 2 \mathrm{H}), 8.06(\mathrm{~d}, J=9.2 \mathrm{~Hz}, 2 \mathrm{H}), 7.99(\mathrm{~d}, J=9.2 \mathrm{~Hz}, 2 \mathrm{H}), 7.91(\mathrm{~s}, 2 \mathrm{H}), 3.24(\mathrm{~s}, 3 \mathrm{H}), 3.10(\mathrm{~s}, 3 \mathrm{H})$, $1.58(\mathrm{~s}, 9 \mathrm{H}) ;{ }^{13} \mathrm{C} \mathrm{NMR}\left(100 \mathrm{MHz}, \mathrm{CDCl}_{3}\right) \quad \delta \quad 155.4,149.1,148.8,132.0,130.6,128.3,126.8,122.7$, 122.6, 122.4, 117.6, 36.8, 36.6, 35.2, 31.9; HRMS (ESI) $\mathrm{m} / \mathrm{z}$ calcd for $\mathrm{C}_{23} \mathrm{H}_{24} \mathrm{NO}_{2}{ }^{+}[\mathrm{M}+\mathrm{H}]^{+}: 346.1802$ found 346.1802 .

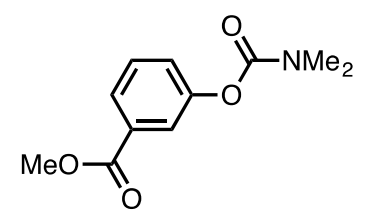

Methyl 3-((dimethylcarbamoyl)oxy)benzoate (1H) 
Method A: Purification by flash silica-gel column chromatography (hexane/EtOAc $=6: 1 \sim 3: 1$ ) afforded $\mathbf{1 H}$ as a colorless liquid (1.09 g, $5.0 \mathrm{mmol}$ scale, $97 \%$ yield). ${ }^{1} \mathrm{H}$ NMR (400 $\mathrm{MHz}, \mathrm{CDCl}_{3}$ ) $\delta$ $7.88(\mathrm{dt}, J=8.0,1.2 \mathrm{~Hz}, 1 \mathrm{H}), 7.79(\mathrm{dd}, J=2.4,1.2 \mathrm{~Hz}, 1 \mathrm{H}), 7.43(\mathrm{t}, J=8.0 \mathrm{~Hz}, 1 \mathrm{H}), 7.33$ (ddd, $J=$ 8.0, 2.4, $1.2 \mathrm{~Hz}, 1 \mathrm{H}), 3.90(\mathrm{~s}, 3 \mathrm{H}), 3.11(\mathrm{~s}, 3 \mathrm{H}), 3.02(\mathrm{~s}, 3 \mathrm{H}) ;{ }^{13} \mathrm{C} \mathrm{NMR}\left(100 \mathrm{MHz}, \mathrm{CDCl}_{3}\right) \quad \delta \quad 166.2$, 154.4, 151.4, 131.3, 129.1, 126.4, 126.3, 122.9, 52.1, 36.6, 36.4; HRMS (ESI) $\mathrm{m} / \mathrm{z}$ calcd for $\mathrm{C}_{11} \mathrm{H}_{13} \mathrm{NO}_{4} \mathrm{Na}^{+}[\mathrm{M}+\mathrm{Na}]^{+}: 246.0737$ found 246.0734 .

\section{2-2. General Procedure for the Synthesis of Aryl Pivalates}

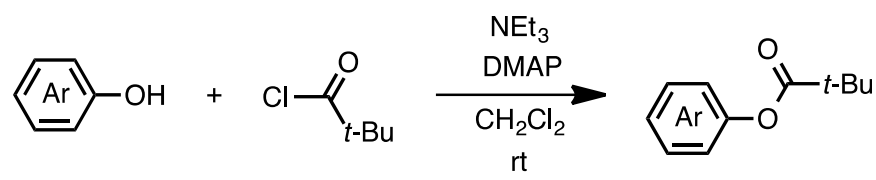

To a solution of the corresponding phenols and a piece of $\mathrm{N}, \mathrm{N}$-dimethyl-4-aminopyridine (DMAP) in dichloroethane $(0.50 \mathrm{M})$ were added $\mathrm{Et}_{3} \mathrm{~N}$ (1.2 equiv) and pivaloyl chloride (1.2 equiv) at $0{ }^{\circ} \mathrm{C}$. This mixture was stirred for 1 hour at room temperature. The reaction mixture was quenched by the addition of saturated $\mathrm{NaHCO}_{3}$ aq, and then the mixture was extracted three times with $\mathrm{CH}_{2} \mathrm{Cl}_{2}$. The combined organic layer was dried over $\mathrm{Na}_{2} \mathrm{SO}_{4}$, and concentrated in vacuo. The residue was purified by flash silica-gel column chromatography to afford the corresponding product.

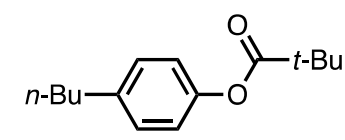

\section{4-Butylphenyl pivalate (1M)}

Purification by flash silica-gel column chromatography (hexane/EtOAc $=40: 1 \sim 10: 1$ ) afforded $\mathbf{1 M}$ as a colorless liquid (1.09 g, $5.0 \mathrm{mmol}$ scale, $93 \%$ yield). ${ }^{1} \mathrm{H} \mathrm{NMR}\left(400 \mathrm{MHz}, \mathrm{CDCl}_{3}\right) \quad \delta \quad 7.16(\mathrm{~d}$, $J=8.8 \mathrm{~Hz}, 2 \mathrm{H}), 6.95(\mathrm{~d}, J=8.8 \mathrm{~Hz}, 2 \mathrm{H}), 2.60(\mathrm{t}, J=8.0 \mathrm{~Hz}, 2 \mathrm{H}), 1.63-1.54(\mathrm{~m}, 2 \mathrm{H}), 1.40-1.30(\mathrm{~m}$, $11 \mathrm{H}), 0.92(\mathrm{t}, J=7.2 \mathrm{~Hz}, 3 \mathrm{H}) ;{ }^{13} \mathrm{C} \mathrm{NMR}\left(100 \mathrm{MHz}, \mathrm{CDCl}_{3}\right) \quad \delta \quad 177.3,148.9,140.1,129.2,121.1$, 39.0, 35.0, 33.6, 27.1, 22.3, 13.9; HRMS (ESI) $m / z$ calcd for $\mathrm{C}_{15} \mathrm{H}_{22} \mathrm{O}_{2} \mathrm{Na}^{+}[\mathrm{M}+\mathrm{Na}]^{+}: 257.1512$ found 257.1511.

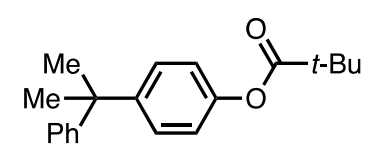

\section{4-(2-Phenylpropan-2-yl)phenyl pivalate (1N)}

Purification by flash silica-gel column chromatography (hexane/EtOAc $=40: 1 \sim 10: 1$ ) afforded $\mathbf{1 N}$ as a colorless liquid (1.50 g, $5.0 \mathrm{mmol} \mathrm{scale}$, quant). ${ }^{1} \mathrm{H}$ NMR (400 $\left.\mathrm{MHz}, \mathrm{CDCl}_{3}\right) \quad \delta \quad 7.29-7.14$ $(\mathrm{m}, 7 \mathrm{H}), 6.95(\mathrm{~d}, J=8.8 \mathrm{~Hz}, 2 \mathrm{H}), 1.67(\mathrm{~s}, 6 \mathrm{H}), 1.34(\mathrm{~s}, 9 \mathrm{H}) ;{ }^{13} \mathrm{C} \mathrm{NMR}\left(100 \mathrm{MHz}, \mathrm{CDCl}_{3}\right) \quad \delta \quad 177.2$, 
150.4, 148.8, 147.9, 128.0, 127.7, 126.7, 125.7, 120.7, 42.7, 39.0, 30.8, 27.1; HRMS (ESI) $\mathrm{m} / \mathrm{z}$ calcd for $\mathrm{C}_{20} \mathrm{H}_{24} \mathrm{O}_{2} \mathrm{Na}^{+}[\mathrm{M}+\mathrm{Na}]^{+}: 319.1669$ found 319.1665 .

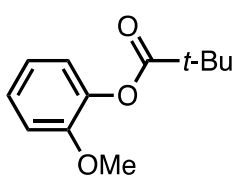

\section{2-Methoxyphenyl pivalate (1R)}

Purification by flash silica-gel column chromatography (hexane/EtOAc $=20: 1 \sim 5: 1$ ) afforded $1 \mathbf{R}$ as a colorless liquid (1.03 g, $5.0 \mathrm{mmol} \mathrm{scale,} \mathrm{99 \%} \mathrm{yield).}{ }^{1} \mathrm{H} \mathrm{NMR}\left(400 \mathrm{MHz}, \mathrm{CDCl}_{3}\right) \quad \delta \quad 7.17$ (ddd, $J=8.4,7.6,2.0 \mathrm{~Hz}, 1 \mathrm{H}), 7.00$ (dd, $J=8.0,2.0 \mathrm{~Hz}, 1 \mathrm{H}), 6.96-6.90(\mathrm{~m}, 2 \mathrm{H}), 3.79$ (s, 3H), 1.37 (s, $9 \mathrm{H}) ;{ }^{13} \mathrm{C}$ NMR $\left(100 \mathrm{MHz}, \mathrm{CDCl}_{3}\right) \quad \delta 176.6,151.2,140.2,126.5,122.7,120.7,112.4,55.8,39.0$, 27.2; HRMS (ESI) $\mathrm{m} / z$ calcd for $\mathrm{C}_{12} \mathrm{H}_{16} \mathrm{O}_{3} \mathrm{Na}^{+}[\mathrm{M}+\mathrm{Na}]^{+}: 231.0992$ found 231.0990.

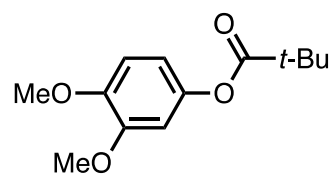

\section{3,4-Dimethoxyphenyl pivalate (1S)}

Purification by flash silica-gel column chromatography (hexane/EtOAc $=20: 1 \sim 6: 1$ ) afforded 1 S as a white solid (1.15 g, $5.0 \mathrm{mmol}$ scale, $97 \%$ yield). ${ }^{1} \mathrm{H}$ NMR $\left(400 \mathrm{MHz}, \mathrm{CDCl}_{3}\right) \quad \delta \quad 6.84(\mathrm{~d}, J=9.2$ $\mathrm{Hz}, 1 \mathrm{H}), 6.62-6.59$ (m, 2H), 3.872 (s, 3H), 3.868 (s, 3H), 1.35 (s, 9H); ${ }^{13} \mathrm{C}$ NMR (100 MHz, $\left.\mathrm{CDCl}_{3}\right)$ $\delta \quad 177.4,149.3,146.6,144.7,112.6,111.0,105.6,56.1,55.9,39.0,27.1$; HRMS (ESI) $\mathrm{m} / \mathrm{z}$ calcd for $\mathrm{C}_{13} \mathrm{H}_{18} \mathrm{O}_{4} \mathrm{Na}^{+}[\mathrm{M}+\mathrm{Na}]^{+}: 261.1097$ found 261.1096.

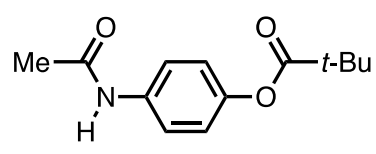

\section{4-Acetamidophenyl pivalate (1V)}

Purification by flash silica-gel column chromatography (hexane/EtOAc $=2: 1 \sim 1: 1$ ) afforded 1V as a white solid $\left(1.03 \mathrm{~g}, 5.0 \mathrm{mmol}\right.$ scale, $87 \%$ yield). ${ }^{1} \mathrm{H}$ NMR $\left(400 \mathrm{MHz}, \mathrm{CDCl}_{3}\right) \quad \delta \quad 7.48(\mathrm{~d}, J=9.2$ $\mathrm{Hz}, 2 \mathrm{H}), 7.00(\mathrm{~d}, J=9.2 \mathrm{~Hz}, 2 \mathrm{H}), 2.16(\mathrm{~s}, 3 \mathrm{H}), 1.35(\mathrm{~s}, 9 \mathrm{H}) ;{ }^{13} \mathrm{C} \mathrm{NMR}\left(100 \mathrm{MHz}, \mathrm{CDCl}_{3}\right) \delta$ 177.6, 168.6, 147.0, 135.6, 121.7, 120.9, 39.0, 27.1, 24.3; HRMS (ESI) $\mathrm{m} / z$ calcd for $\mathrm{C}_{13} \mathrm{H}_{17} \mathrm{NO}_{3} \mathrm{Na}^{+}[\mathrm{M}+$ $\mathrm{Na}^{+}: 258.1101$ found 258.1099.<smiles>CC(C)(C)C(=O)Oc1ccc2oc(-c3ccccc3)cc(=O)c2c1</smiles> 


\section{4-Oxo-2-phenyl-4H-chromen-6-yl pivalate (1X)}

Purification by flash silica-gel column chromatography (hexane/EtOAc $=10: 1 \sim 3: 1$ ) afforded $\mathbf{1 X}$ as a white solid (965.8 mg, $3.0 \mathrm{mmol}$ scale, quant). ${ }^{1} \mathrm{H} \mathrm{NMR}\left(400 \mathrm{MHz}, \mathrm{CDCl}_{3}\right) \quad \delta$ 7.95-7.91 (m, $2 \mathrm{H}), 7.89(\mathrm{~d}, J=2.8 \mathrm{~Hz}, 1 \mathrm{H}), 7.61(\mathrm{~d}, J=9.2 \mathrm{~Hz}, 1 \mathrm{H}), 7.57-7.51(\mathrm{~m}, 3 \mathrm{H}), 7.42(\mathrm{dd}, J=9.2,2.8 \mathrm{~Hz}$, 1H), 6.83 (s, 1H), 1.39 (s, 9H); ${ }^{13} \mathrm{C}$ NMR (100 MHz, $\left.\mathrm{CDCl}_{3}\right) \quad \delta \quad 177.8$ 177.0, 163.6, 153.6, 148.1, 131.7, 131.6, 129.1, 128.0, 126.3, 124.6, 119.3, 117.6, 107.1, 39.1, 27.1; HRMS (ESI) $\mathrm{m} / \mathrm{z}$ calcd for $\mathrm{C}_{20} \mathrm{H}_{18} \mathrm{O}_{4} \mathrm{Na}^{+}[\mathrm{M}+\mathrm{Na}]^{+}: 345.1097$ found 345.1096.<smiles>COC(=O)C(Cc1ccc(OC(=O)[18F])cc1)NP</smiles>

\section{(S)-4-(2-(1,3-Dioxoisoindolin-2-yl)-3-methoxy-3-oxopropyl)phenyl pivalate (1Z)}

Purification by flash silica-gel column chromatography (hexane/EtOAc $=10: 1 \sim 2: 1$ ) afforded 1Z as a white solid (1.50 g, $4.0 \mathrm{mmol}$ scale, $91 \%$ yield). ${ }^{1} \mathrm{H}$ NMR $\left(400 \mathrm{MHz}, \mathrm{CDCl}_{3}\right) \quad \delta \quad 7.79$ (d, $J=5.6$, $3.2 \mathrm{~Hz}, 2 \mathrm{H}), 7.70(\mathrm{dd}, J=5.6,3.2 \mathrm{~Hz}, 2 \mathrm{H}), 7.17(\mathrm{~d}, J=8.8 \mathrm{~Hz}, 2 \mathrm{H}), 6.89$ (d, $J=8.8 \mathrm{~Hz}, 2 \mathrm{H}), 5.15$ (dd, $J=10.8,6.0 \mathrm{~Hz}, 1 \mathrm{H}), 3.78(\mathrm{~s}, 3 \mathrm{H}), 3.60-3.54(\mathrm{~m}, 2 \mathrm{H}), 1.30(\mathrm{~s}, 9 \mathrm{H}),{ }^{13} \mathrm{C}$ NMR $\left(100 \mathrm{MHz}, \mathrm{CDCl}_{3}\right) \quad \delta$ 176.9, 169.2, 167.5, 149.8, 134.1, 133.9, 131.5, 129.7, 123.5, 121.6, 53.1, 52.9, 39.0, 34.0, 27.0; HRMS (ESI) $m / z$ calcd for $\mathrm{C}_{23} \mathrm{H}_{23} \mathrm{NO}_{6} \mathrm{Na}^{+}[\mathrm{M}+\mathrm{Na}]^{+}: 432.1418$ found 432.1422 .

\section{2-3. The Synthesis of 5-methoxy-3,4-dihydronaphthalen-2-yl dimethylcarbamate (1AC):}
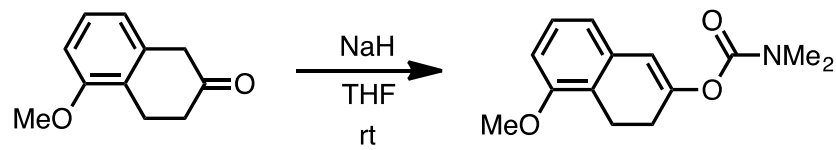

$\mathrm{NaH}$ (60\% dispersion in paraffin liquid: $360.0 \mathrm{mg}, 9.0 \mathrm{mmol}, 1.8$ equiv) was placed in a 100-mL 2-necked flask under a stream of argon, and THF $(5.0 \mathrm{~mL})$ was added to the flask. To this suspension, 5-methoxy-3,4-dihydronaphthalen-2(1H)-one $(881.1 \mathrm{mg}, 5.0 \mathrm{mmol}, 1.0$ equiv) in THF (5.0 mL) was added dropwise. The resulting mixture was stirred at room temperature for $5 \mathrm{~min}$. A solution of $N, N$-dimethylcarbamoyl chloride $(0.69 \mathrm{~mL}, 7.5 \mathrm{mmol}, 1.5$ equiv) in THF (2.0 mL) was added, and then the mixture was stirred for an additional $30 \mathrm{~min}$. The reaction was quenched by the addition of water. The mixture was extracted three times with diethyl ether, and the combined organic layer was washed with water and brine, dried over $\mathrm{Na}_{2} \mathrm{SO}_{4}$, and then filtered. The filtrate was concentrated in vacuo. The crude residue was purified by flash silica-gel column chromatography (hexane/EtOAc $=$ 10:1 4:1) to afford $\mathbf{1 A C}$ as a pale yellow liquid (1.05 g, 84\% yield). ${ }^{1} \mathrm{H} \mathrm{NMR}\left(400 \mathrm{MHz}, \mathrm{CDCl}_{3}\right): \delta$ $7.10(\mathrm{dd}, J=8.4,7.6 \mathrm{~Hz}, 1 \mathrm{H}), 6.72(\mathrm{~d}, J=8.4 \mathrm{~Hz}, 1 \mathrm{H}), 6.65(\mathrm{~d}, J=7.6 \mathrm{~Hz}, 1 \mathrm{H}), 6.18(\mathrm{~s}, 1 \mathrm{H}), 3.82(\mathrm{~s}$, $3 \mathrm{H}), 3.03-2.95(\mathrm{~m}, 8 \mathrm{H}), 2.51(\mathrm{t}, J=8.8 \mathrm{~Hz}, 2 \mathrm{H}) ;{ }^{13} \mathrm{C} \mathrm{NMR}\left(100 \mathrm{MHz}, \mathrm{CDCl}_{3}\right): \delta 156.1,154.3,151.7$, 
134.9, 126.9, 120.7, 119.0, 113.7, 109.2, 55.4, 36.5, 36.4, 26.0, 21.1; HRMS (ESI) $\mathrm{m} / \mathrm{z}$ calcd for $\mathrm{C}_{14} \mathrm{H}_{17} \mathrm{NO}_{3} \mathrm{Na}^{+}[\mathrm{M}+\mathrm{Na}]^{+}: 270.1101$ found 270.1100 .

\section{Ni-Catalyzed Cyanation of Aryl Carbamates with Aminoacetonitrile 2}

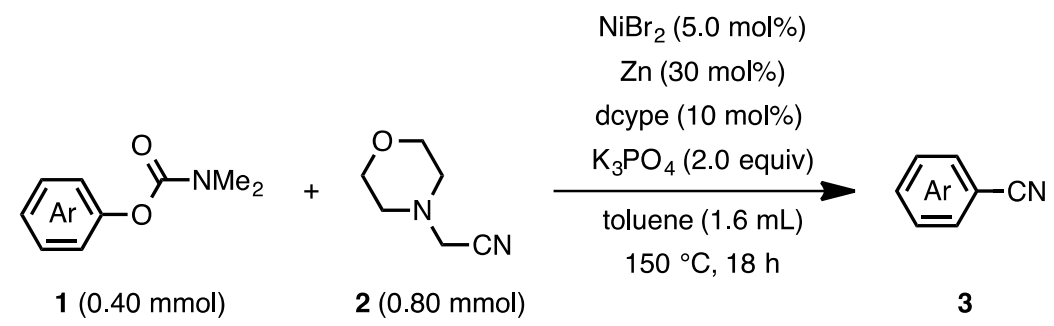

General Procedure: A 20-mL glass vessel equipped with a J. Young® O-ring tap containing a magnetic stirring bar and $\mathrm{K}_{3} \mathrm{PO}_{4}(169.8 \mathrm{mg}, 0.80 \mathrm{mmol}, 2.0$ equiv) and $\mathrm{Zn}$ powder $(7.9 \mathrm{mg}, 0.12 \mathrm{mmol}$, $30 \mathrm{~mol} \%$ ) were dried with a heatgun for $3 \mathrm{~min}$ in vacuo and filled with $\mathrm{N}_{2}$ after cooling to room temperature. To this vessel were added $\mathrm{NiBr}_{2}(4.4 \mathrm{mg}, 0.020 \mathrm{mmol}, 5.0 \mathrm{~mol} \%)$, phenol derivatives 1 ( $0.40 \mathrm{mmol}, 1.0$ equiv) and 2-morpholinoacetonitrile 2 (100.9 mg, $0.80 \mathrm{mmol}, 2.0$ equiv). After the vessel was introduced into an argon-atmosphere glovebox, to the reaction vessel was added 1,2-bis(dicyclohexylphosphino)ethane (dcype: $16.9 \mathrm{mg}, 0.040 \mathrm{mmol}, 10 \mathrm{~mol} \%$ ) and dry toluene (1.6 $\mathrm{mL}$ ), and then the vessel was taken out of the glovebox. The vessel was sealed with an O-ring tap and then heated at $150{ }^{\circ} \mathrm{C}$ for $18 \mathrm{~h}$ in an 8 -well reaction block with stirring. After cooling the reaction mixture to room temperature, the mixture was passed through a silica gel pad with EtOAc as the eluent. The filtrate was concentrated and the residue was subjected to PTLC, flash silica-gel column chromatography or GPC to afford aryl nitriles 3.

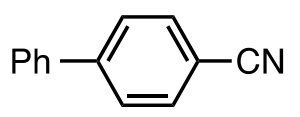

\section{[1,1'-Biphenyl]-4-carbonitrile (3A) ${ }^{[7]}$}

Purification by PTLC (hexane/EtOAc $=4: 1)$ afforded $3 \mathrm{~A}$ as a white solid $(61.7 \mathrm{mg}, 86 \%$ yield). ${ }^{1} \mathrm{H}$ NMR $\left(400 \mathrm{MHz}, \mathrm{CDCl}_{3}\right) \quad \delta \quad 7.76-7.66(\mathrm{~m}, 4 \mathrm{H}), 7.62-7.56(\mathrm{~m}, 2 \mathrm{H}), 7.52-7.40(\mathrm{~m}, 3 \mathrm{H}) ;{ }^{13} \mathrm{C} \mathrm{NMR}$ $\left(100 \mathrm{MHz}, \mathrm{CDCl}_{3}\right) \quad \delta \quad 145.6,139.1,132.6,129.1,128.6,127.7,127.2,118.9,110.9 ;$ HRMS (APCI) $m / z$ calcd for $\mathrm{C}_{13} \mathrm{H}_{10} \mathrm{~N}^{+}[\mathrm{M}+\mathrm{H}]^{+}: 180.0808$ found 180.0809 .

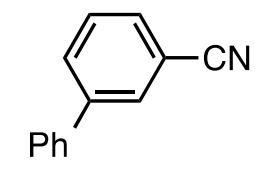

$\left[1,1^{\prime}\right.$-Biphenyl]-3-carbonitrile (3B) ${ }^{[8]}$

[7] J. Kim, J. Choi, K. Shin, S. Chang, J. Am. Chem. Soc. 2012, 134, 2528.

[8] W.-J. Zhou, K.-H. Wang, J.-X. Wang, J. Org. Chem. 2009, 74, 5599. 
Purification by PTLC (hexane/EtOAc $=4: 1$ ) afforded 3B as a white solid ( $28.5 \mathrm{mg}, 40 \%$ yield). ${ }^{1} \mathrm{H}$ NMR $\left(400 \mathrm{MHz}, \mathrm{CDCl}_{3}\right) \quad \delta$ 7.86-7.84 (m, 1H), $7.81(\mathrm{ddd}, J=8.0,2.0,1.2 \mathrm{~Hz}, 1 \mathrm{H}), 7.62(\mathrm{dt}, J=$ 8.0, $1.2 \mathrm{~Hz}, 1 \mathrm{H}), 7.57-7.53(\mathrm{~m}, 3 \mathrm{H}), 7.52-7.38(\mathrm{~m}, 3 \mathrm{H}) ;{ }^{13} \mathrm{C} \mathrm{NMR}\left(100 \mathrm{MHz}, \mathrm{CDCl}_{3}\right) \quad \delta \quad 142.4$, $138.8,131.4,130.6,129.6,129.1,128.4,127.0,118.8,112.9$ (one peak is overlapping); HRMS (APCI) $\mathrm{m} / \mathrm{z}$ calcd for $\mathrm{C}_{13} \mathrm{H}_{10} \mathrm{~N}^{+}[\mathrm{M}+\mathrm{H}]^{+}: 180.0808$ found 180.0809 .

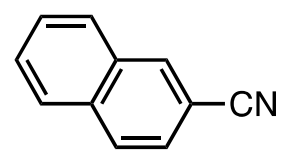

\section{2-Naphthonitrile (3C) ${ }^{[7]}$}

Purification by PTLC (hexane/EtOAc $=4: 1$ ) afforded $3 \mathrm{C}$ as a yellow solid (49.6 $\mathrm{mg}, 81 \%$ yield). ${ }^{1} \mathrm{H}$ NMR $\left(400 \mathrm{MHz}, \mathrm{CDCl}_{3}\right) \quad \delta \quad 8.21(\mathrm{~s}, 1 \mathrm{H}), 7.92-7.85(\mathrm{~m}, 3 \mathrm{H}), 7.67-7.85(\mathrm{~m}, 3 \mathrm{H}) ;{ }^{13} \mathrm{C}$ NMR $(100$ $\left.\mathrm{MHz}, \mathrm{CDCl}_{3}\right) \quad \delta \quad 134.6,134.1,132.2,129.1,129.0,128.3,128.0,127.6,126.3,119.2,109.3$; HRMS (APCI) $m / z$ calcd for $\mathrm{C}_{11} \mathrm{H}_{8} \mathrm{~N}^{+}[\mathrm{M}+\mathrm{H}]^{+}: 154.0651$ found 154.0653 .

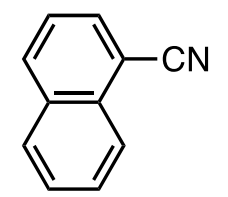

\section{1-Naphthonitrile (3D) ${ }^{[7]}$}

Purification by PTLC (hexane/EtOAc $=4: 1$ ) afforded 3D as a white solid (43.9 $\mathrm{mg}, 72 \%$ yield). ${ }^{1} \mathrm{H}$ NMR $\left(400 \mathrm{MHz}, \mathrm{CDCl}_{3}\right) \quad \delta \quad 8.22(\mathrm{~d}, J=8.4 \mathrm{~Hz}, 1 \mathrm{H}), 8.06(\mathrm{~d}, J=8.4 \mathrm{~Hz}, 1 \mathrm{H}), 7.93-7.87(\mathrm{~m}, 2 \mathrm{H})$, $7.68(\mathrm{t}, J=8.0 \mathrm{~Hz}, 1 \mathrm{H}), 7.61(\mathrm{t}, J=8.0 \mathrm{~Hz}, 1 \mathrm{H}), 7.51(\mathrm{dd}, J=8.4,7.6 \mathrm{~Hz}, 1 \mathrm{H}) ;{ }^{13} \mathrm{C} \mathrm{NMR}(100 \mathrm{MHz}$, $\left.\mathrm{CDCl}_{3}\right) \delta 133.2,132.9,132.2,129.1,129.0,128.3,128.0,127.6,126.3,119.2,109.3$; HRMS (APCI) $m / z$ calcd for $\mathrm{C}_{11} \mathrm{H}_{8} \mathrm{~N}^{+}[\mathrm{M}+\mathrm{H}]^{+}: 154.0651$ found 154.0652 .

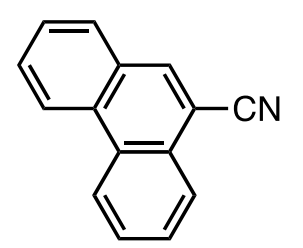

Phenanthrene-9-carbonitrile (3E) ${ }^{[7]}$

Purification by PTLC (hexane/EtOAc $=4: 1$ ) afforded $\mathbf{3 E}$ as a white solid (47.7 $\mathrm{mg}, 59 \%$ yield). ${ }^{1} \mathrm{H}$ NMR $\left(400 \mathrm{MHz}, \mathrm{CDCl}_{3}\right) \quad \delta \quad 8.66-8.59(\mathrm{~m}, 2 \mathrm{H}), 8.27-8.22(\mathrm{~m}, 1 \mathrm{H}), 8.16(\mathrm{~s}, 1 \mathrm{H}), 7.86(\mathrm{~d}, J=8.0$ $\mathrm{Hz}, 1 \mathrm{H}), 7.78-7.68(\mathrm{~m}, 3 \mathrm{H}), 7.64(\mathrm{t}, J=8.0 \mathrm{~Hz}, 1 \mathrm{H}) ;{ }^{13} \mathrm{C} \mathrm{NMR}\left(100 \mathrm{MHz}, \mathrm{CDCl}_{3}\right) \quad \delta \quad 135.5,131.6$, $129.9,129.7,129.6,129.4,128.7,128.1,128.0,127.5,126.0,123.0,122.8,117.8,109.3$; HRMS (APCI) $m / z$ calcd for $\mathrm{C}_{15} \mathrm{H}_{10} \mathrm{~N}^{+}[\mathrm{M}+\mathrm{H}]^{+}: 204.0808$ found 204.0809 .

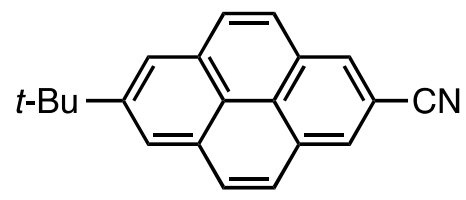




\section{7-(tert-Butyl)pyrene-2-carbonitrile (3F)}

Purification by GPC afforded $\mathbf{3 F}$ as a white solid (63.5 mg, 56\% yield). ${ }^{1} \mathrm{H}$ NMR (400 MHz, $\left.\mathrm{CDCl}_{3}\right) \delta 8.24(\mathrm{~s}, 2 \mathrm{H}), 8.19(\mathrm{~s}, 2 \mathrm{H}), 8.03(\mathrm{~d}, J=9.2 \mathrm{~Hz}, 2 \mathrm{H}), 7.86(\mathrm{~d}, J=9.2 \mathrm{~Hz}, 2 \mathrm{H}), 1.59(\mathrm{~s}, 9 \mathrm{H})$; ${ }^{13} \mathrm{C}$ NMR $\left(100 \mathrm{MHz}, \mathrm{CDCl}_{3}\right) \quad \delta \quad 150.9,131.3,130.8,129.2,127.0,126.2,125.8,123.3,121.8,119.7$, 108.4, 35.4, 31.8; HRMS (APCI) $m / z$ calcd for $\mathrm{C}_{21} \mathrm{H}_{18} \mathrm{~N}^{+}[\mathrm{M}+\mathrm{H}]^{+}: 284.1434$ found 284.1434.

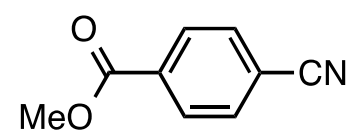

\section{Methyl 4-cyanobenzoate (3G) ${ }^{[9]}$}

Purification by flash silica-gel column chromatography (hexane/EtOAc $=20: 1 \sim 7: 1$ ) afforded $3 \mathbf{G}$ as a white solid (35.3 mg, 55\% yield). ${ }^{1} \mathrm{H}$ NMR (400 MHz, $\left.\mathrm{CDCl}_{3}\right) \quad \delta \quad 8.14(\mathrm{~d}, J=8.8 \mathrm{~Hz}, 2 \mathrm{H})$, $7.75(\mathrm{~d}, J=8.8 \mathrm{~Hz}, 2 \mathrm{H}), 3.97(\mathrm{~s}, 3 \mathrm{H}) ;{ }^{13} \mathrm{C} \mathrm{NMR}\left(100 \mathrm{MHz}, \mathrm{CDCl}_{3}\right) \quad \delta \quad 165.4,133.9,132.2,130.0$, 117.9, 116.3, 52.7; HRMS (APCI) $m / z$ calcd for $\mathrm{C}_{9} \mathrm{H}_{8} \mathrm{NO}_{2}{ }^{+}[\mathrm{M}+\mathrm{H}]^{+}: 162.0550$ found 162.0551 .

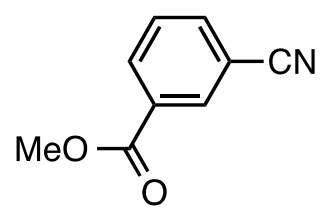

\section{Methyl 3-cyanobenzoate (3H) ${ }^{[10]}$}

Purification by PTLC (hexane/EtOAc $=4: 1$ ) afforded $\mathbf{3 H}$ as a white solid (36.1 $\mathrm{mg}, 56 \%$ yield). ${ }^{1} \mathrm{H}$ NMR (400 MHz, $\left.\mathrm{CDCl}_{3}\right) \quad \delta \quad 8.33(\mathrm{t}, J=1.6 \mathrm{~Hz}, 1 \mathrm{H}), 8.27(\mathrm{dt}, J=8.0,1.2 \mathrm{~Hz}, 1 \mathrm{H}), 7.85(\mathrm{td}, J=$ 8.0, $1.6 \mathrm{~Hz}, 1 \mathrm{H}), 7.60(\mathrm{t}, J=8.0 \mathrm{~Hz}, 1 \mathrm{H}), 3.97(\mathrm{~s}, 3 \mathrm{H}) ;{ }^{13} \mathrm{C} \mathrm{NMR}\left(100 \mathrm{MHz}, \mathrm{CDCl}_{3}\right) \quad \delta \quad 165.0,135.9$, 133.6, 133.2, 131.4, 129.4, 117.8, 112.9, 52.7; HRMS (APCI) $\mathrm{m} / z$ calcd for $\mathrm{C}_{9} \mathrm{H}_{8} \mathrm{NO}_{2}{ }^{+}[\mathrm{M}+\mathrm{H}]^{+}$: 162.0550 found 162.0551 .

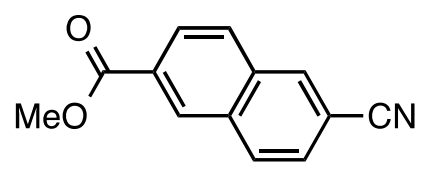

\section{Methyl 6-cyano-2-naphthoate (3I)}

Purification by GPC afforded 3I as a white solid (46.3 mg, 55\% yield). ${ }^{1} \mathrm{H}$ NMR (400 MHz, $\left.\mathrm{CDCl}_{3}\right) \delta 8.63(\mathrm{~s}, 1 \mathrm{H}), 8.27(\mathrm{~s}, 1 \mathrm{H}), 8.18(\mathrm{dd}, J=8.4,1.6 \mathrm{~Hz}, 1 \mathrm{H}), 8.04(\mathrm{~d}, J=8.8 \mathrm{~Hz}, 1 \mathrm{H}), 7.95(\mathrm{~d}$, $J=8.8 \mathrm{~Hz}, 1 \mathrm{H}), 7.68(\mathrm{dd}, J=8.4,1.6 \mathrm{~Hz}, 1 \mathrm{H}), 4.01(\mathrm{~s}, 3 \mathrm{H}) ;{ }^{13} \mathrm{C} \mathrm{NMR}\left(100 \mathrm{MHz}, \mathrm{CDCl}_{3}\right) \quad \delta \quad 166.3$, 134.1, 133.8, 130.7, 130.5, 130.3, 128.7, 127.1, 127.0, 118.7, 111.6, 52.5 (one peak is overlapping); HRMS (APCI) $m / z$ calcd for $\mathrm{C}_{13} \mathrm{H}_{10} \mathrm{NO}_{2}{ }^{+}[\mathrm{M}+\mathrm{H}]^{+}: 212.0706$ found 212.0707.

[9] R. S. Jensen, A. S. Gajare, K. Toyota, M. Yoshifuji, F. Ozawa, Tetrahedron Lett. 2005, 46, 8645.

[10] P. Y. Yeung, C. M. So, C. P. Lau, F. Y. Kwong, Angew. Chem. Int. Ed. 2010, 49, 8918. 


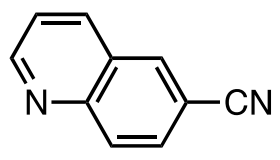

Quinoline-6-carbonitrile (3J) ${ }^{[10]}$

Purification by PTLC (hexane/EtOAc $=1: 1$ ) afforded $\mathbf{3 J}$ as a white solid (30.3 $\mathrm{mg}, 49 \%$ yield). ${ }^{1} \mathrm{H}$ NMR (400 MHz, $\left.\mathrm{CDCl}_{3}\right) \quad \delta 9.07(\mathrm{dd}, J=4.4,2.0 \mathrm{~Hz}, 1 \mathrm{H}), 8.28-8.18(\mathrm{~m}, 3 \mathrm{H}), 7.87$ (dd, $J=8.8$, $2.0 \mathrm{~Hz}, 1 \mathrm{H}), 7.55(\mathrm{dd}, J=8.8,4.4 \mathrm{~Hz}, 1 \mathrm{H}) ;{ }^{13} \mathrm{C} \mathrm{NMR}\left(100 \mathrm{MHz}, \mathrm{CDCl}_{3}\right) \quad \delta \quad 153.2,149.1,136.3$, 134.1, 131.0, 130.1, 127.5, 122.7, 118.5, 110.3; HRMS (APCI) $\mathrm{m} / z$ calcd for $\mathrm{C}_{10} \mathrm{H}_{7} \mathrm{~N}_{2}{ }^{+}[\mathrm{M}+\mathrm{H}]^{+}$: 155.0604 found 155.0604 .

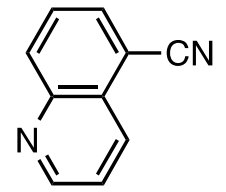

\section{Quinoline-5-carbonitrile (3K)}

Purification by PTLC (hexane/EtOAc = 2:3) afforded 3K as a white solid (26.1 $\mathrm{mg}, 42 \%$ yield). ${ }^{1} \mathrm{H}$ NMR $\left(400 \mathrm{MHz}, \mathrm{CDCl}_{3}\right) \quad \delta 9.06(\mathrm{dd}, J=4.4,1.6 \mathrm{~Hz}, 1 \mathrm{H}), 8.56(\mathrm{~d}, J=8.4 \mathrm{~Hz}, 1 \mathrm{H}), 8.37(\mathrm{~d}, J=$ $8.4 \mathrm{~Hz}, 1 \mathrm{H}), 8.00(\mathrm{dd}, J=7.2,1.8 \mathrm{~Hz}, 1 \mathrm{H}), 7.79(\mathrm{dd}, J=8.8,7.2 \mathrm{~Hz}, 1 \mathrm{H}), 7.63(\mathrm{dd}, J=8.8,4.4 \mathrm{~Hz}$, $1 \mathrm{H}) ;{ }^{13} \mathrm{C}$ NMR $\left(100 \mathrm{MHz}, \mathrm{CDCl}_{3}\right) \quad \delta \quad 152.0,147.5,135.1,133.3,133.0,128.6,127.9,123.2,116.6$, 110.3; HRMS (APCI) $m / z$ calcd for $\mathrm{C}_{10} \mathrm{H}_{7} \mathrm{~N}_{2}{ }^{+}[\mathrm{M}+\mathrm{H}]^{+}$: 155.0604 found 155.0603.

\section{Ni-Catalyzed Cyanation of Aryl Pivalates with Aminoacetonitrile 2}

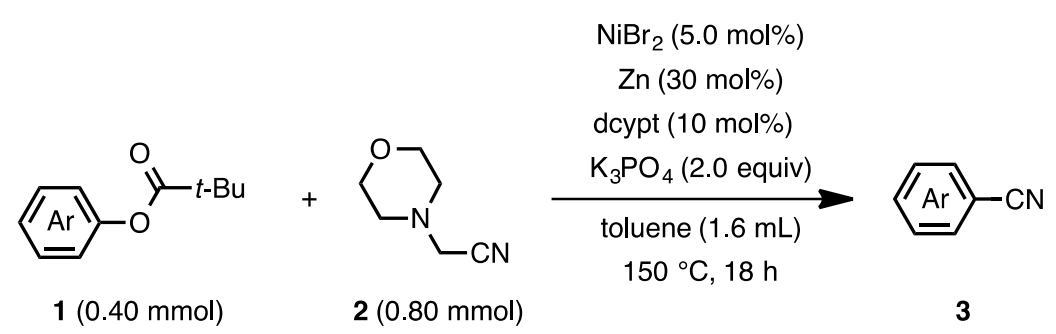

General Procedure: A 20-mL glass vessel equipped with a J. Young® O-ring tap containing a magnetic stirring bar and $\mathrm{K}_{3} \mathrm{PO}_{4}(169.8 \mathrm{mg}, 0.80 \mathrm{mmol}, 2.0$ equiv) and $\mathrm{Zn}$ powder $(7.9 \mathrm{mg}, 0.12 \mathrm{mmol}$, $30 \mathrm{~mol} \%$ ) were dried with a heatgun for $3 \mathrm{~min}$ in vacuo and filled with $\mathrm{N}_{2}$ after cooling to room temperature. To this vessel were added $\mathrm{NiBr}_{2}(4.4 \mathrm{mg}, 0.020 \mathrm{mmol}, 5.0 \mathrm{~mol} \%)$, phenol derivatives 1 (0.40 mmol, 1.0 equiv), 2-morpholinoacetonitrile 2 (100.9 mg, $0.80 \mathrm{mmol}, 2.0$ equiv) and 3,4-bis(dicyclohexylphosphino)thiophene (dcypt: $19.1 \mathrm{mg}, 0.040 \mathrm{mmol}, 10 \mathrm{~mol} \%$ ). Then, dry toluene $(1.6 \mathrm{~mL})$ was added under a stream of $\mathrm{N}_{2}$. The vessel was sealed with an O-ring tap and then heated at $150{ }^{\circ} \mathrm{C}$ for $18 \mathrm{~h}$ in an 8 -well reaction block with stirring. After cooling the reaction mixture to room temperature, the mixture was passed through a silica gel pad with EtOAc as the eluent. The filtrate 
was concentrated and the residue was subjected to PTLC, flash silica-gel column chromatography or GPC to afford aryl nitriles 3 .

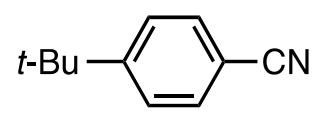

\section{4-(tert-Butyl)benzonitrile (3L) $)^{[9]}$}

Purification by GPC afforded 3L as a white solid (41.2 mg, 65\% yield). ${ }^{1} \mathrm{H}$ NMR (600 MHz, $\left.\mathrm{CDCl}_{3}\right) \quad \delta \quad 7.79(\mathrm{~d}, J=8.4 \mathrm{~Hz}, 2 \mathrm{H}), 7.48(\mathrm{~d}, J=8.4 \mathrm{~Hz}, 2 \mathrm{H}), 1.33(\mathrm{~s}, 9 \mathrm{H}) ;{ }^{13} \mathrm{C}$ NMR $(150 \mathrm{MHz}$, $\left.\mathrm{CDCl}_{3}\right) \quad \delta \quad 156.6,131.9,126.1,119.1,109.2,35.2,30.9$; HRMS (APCI) $\mathrm{m} / z$ calcd for $\mathrm{C}_{11} \mathrm{H}_{14} \mathrm{~N}^{+}[\mathrm{M}+$ $\mathrm{H}]^{+}: 160.1121$ found 160.1122 .

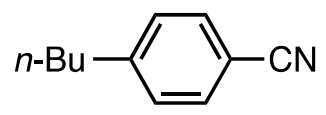

\section{4-Butylbenzonitrile (3M) ${ }^{[8]}$}

Purification by PTLC ( hexane/EtOAc $=8: 1)$ afforded $\mathbf{3 M}$ as a colorless liquid $(41.8 \mathrm{mg}, 66 \%$ yield). ${ }^{1} \mathrm{H} \mathrm{NMR}\left(400 \mathrm{MHz}, \mathrm{CDCl}_{3}\right) \quad \delta \quad 7.56(\mathrm{~d}, J=8.4 \mathrm{~Hz}, 2 \mathrm{H}), 7.27(\mathrm{~d}, J=8.4 \mathrm{~Hz}, 2 \mathrm{H}), 2.67(\mathrm{t}, J=$ $8.0 \mathrm{~Hz}, 2 \mathrm{H}), 1.65-1.56(\mathrm{~m}, 2 \mathrm{H}), 1.35(\mathrm{se}, J=7.6 \mathrm{~Hz}, 2 \mathrm{H}), 0.93(\mathrm{t}, J=7.2 \mathrm{~Hz}, 3 \mathrm{H}) ;{ }^{13} \mathrm{C}$ NMR $(100$ $\left.\mathrm{MHz}, \mathrm{CDCl}_{3}\right) \delta \quad 148.6,132.1,129.2,119.2,109.4,35.8,33.1,22.2,13.8$; HRMS (APCI) $\mathrm{m} / \mathrm{z}$ calcd for $\mathrm{C}_{11} \mathrm{H}_{14} \mathrm{~N}^{+}[\mathrm{M}+\mathrm{H}]^{+}: 160.1121$ found 160.1122 .

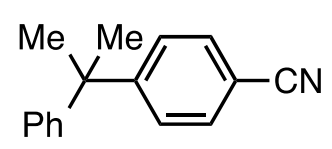

\section{4-(2-Phenylpropan-2-yl)benzonitrile (3N $)^{[11]}$}

Purification by PTLC (hexane/EtOAc $=8: 1)$ afforded $\mathbf{3 N}$ as a colorless liquid $(69.3 \mathrm{mg}, 78 \%$ yield). ${ }^{1} \mathrm{H}$ NMR (400 MHz, $\left.\mathrm{CDCl}_{3}\right) \quad \delta \quad 7.54(\mathrm{~d}, J=8.4 \mathrm{~Hz}, 2 \mathrm{H}), 7.34-7.25$ (m, $\left.4 \mathrm{H}\right), 7.22-7.16$ (m, $3 \mathrm{H}), 1.68$ (s, 6H); ${ }^{13} \mathrm{C} \mathrm{NMR}\left(100 \mathrm{MHz}, \mathrm{CDCl}_{3}\right) \quad \delta$ 156.2, 149.0, 131.8, 128.2, 127.6, 126.6, 126.1, 119.0, 109.5, 43.4, 30.3; HRMS (APCI) $m / z$ calcd for $\mathrm{C}_{16} \mathrm{H}_{16} \mathrm{~N}^{+}[\mathrm{M}+\mathrm{H}]^{+}: 222.1277$ found 222.1278.

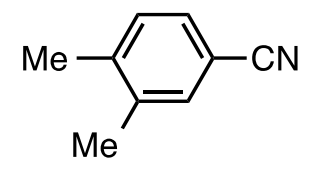

\section{3,4-Dimethylbenzonitrile (30) ${ }^{[12]}$}

Purification by PTLC (hexane/EtOAc $=9: 1$ ) afforded 30 as a white solid (43.2 $\mathrm{mg}, 82 \%$ yield). ${ }^{1} \mathrm{H}$ NMR (400 MHz, $\left.\mathrm{CDCl}_{3}\right) \quad \delta$ 7.41-7.35 (m, 2H), $7.21(\mathrm{~d}, J=8.0 \mathrm{~Hz}, 1 \mathrm{H}), 2.32(\mathrm{~s}, 3 \mathrm{H}), 2.28(\mathrm{~s}$, $3 \mathrm{H}) ;{ }^{13} \mathrm{C}$ NMR $\left(100 \mathrm{MHz}, \mathrm{CDCl}_{3}\right) \quad \delta \quad 142.4,137.8,132.8,130.2,129.6,119.2,109.4,20.1,19.5$; HRMS (APCI) $m / z$ calcd for $\mathrm{C}_{9} \mathrm{H}_{10} \mathrm{~N}^{+}[\mathrm{M}+\mathrm{H}]^{+}: 132.0808$ found 132.0810.

[11] P. Maslak, W. H. Chapman, Jr., Tetrahedron 1990, 46, 2715.

[12] C. W. Liskey, X. Liao, J. F. Hartwig, J. Am. Chem. Soc. 2010, 132, 11389. 


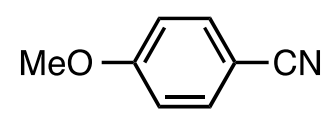

\section{4-Methoxybenzonitrile (3P) ${ }^{[7]}$}

Purification by flash silica-gel column chromatography (hexane/EtOAc $=10: 1 \sim 5: 1$ ) afforded 3P as a white solid (46.5 mg, 87\% yield). ${ }^{1} \mathrm{H}$ NMR (400 MHz, $\left.\mathrm{CDCl}_{3}\right) \quad \delta 7.59(\mathrm{~d}, J=9.2 \mathrm{~Hz}, 2 \mathrm{H}), 6.95$ $(\mathrm{d}, J=9.2 \mathrm{~Hz}, 2 \mathrm{H}), 3.86(\mathrm{~s}, 3 \mathrm{H}) ;{ }^{13} \mathrm{C} \mathrm{NMR}\left(100 \mathrm{MHz}, \mathrm{CDCl}_{3}\right) \quad \delta \quad 162.8,133.9,119.2,114.7,103.9$, 55.5; HRMS (APCI) $m / z$ calcd for $\mathrm{C}_{8} \mathrm{H}_{8} \mathrm{NO}^{+}[\mathrm{M}+\mathrm{H}]^{+}: 134.0600$ found 134.0602 .

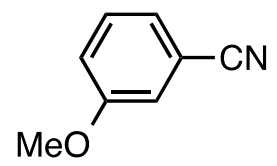

\section{3-Methoxybenzonitrile (3Q) ${ }^{[7]}$}

The reaction was conducted using $\mathrm{NiBr}_{2}(8.7 \mathrm{mg}, 0.040 \mathrm{mmol}, 10 \mathrm{~mol} \%)$, dcypt (38.1 mg, 0.080 mmol, $20 \mathrm{~mol} \%$ ) and $\mathrm{Zn}$ powder (15.7 $\mathrm{mg}, 0.24 \mathrm{mmol}, 60 \mathrm{~mol} \%)$. Purification by PTLC (hexane/EtOAc $=4: 1)$ afforded $\mathbf{3 Q}$ as a colorless liquid $\left(34.7 \mathrm{mg}, 65 \%\right.$ yield). ${ }^{1} \mathrm{H}$ NMR (400 MHz, $\left.\mathrm{CDCl}_{3}\right) \quad \delta \quad 7.40-7.35(\mathrm{~m}, 1 \mathrm{H}), 7.26-7.23(\mathrm{~m}, 1 \mathrm{H}), 7.16-7.11(\mathrm{~m}, 2 \mathrm{H}), 3.84(\mathrm{~s}, 3 \mathrm{H}) ;{ }^{13} \mathrm{C}$ NMR $(100$ $\left.\mathrm{MHz}, \mathrm{CDCl}_{3}\right) \delta \quad 159.6,130.3,124.5,119.3,118.7,116.8,113.2,55.5$; HRMS (APCI) $\mathrm{m} / \mathrm{z}$ calcd for $\mathrm{C}_{8} \mathrm{H}_{8} \mathrm{NO}^{+}[\mathrm{M}+\mathrm{H}]^{+}: 134.0600$ found 134.0602.

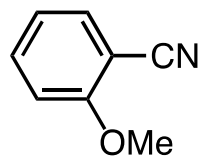

\section{2-Methoxybenzonitrile (3R) ${ }^{[7]}$}

Purification by PTLC (hexane/EtOAc $=7: 1)$ afforded $3 \mathbf{R}$ as a colorless solid $(37.1 \mathrm{mg}, 70 \%$ yield). ${ }^{1} \mathrm{H}$ NMR (400 MHz, $\left.\mathrm{CDCl}_{3}\right) \quad \delta \quad 7.58-7.51$ (m, 2H), 7.04-6.96 (m, 2H), $3.93(\mathrm{~s}, 3 \mathrm{H}) ;{ }^{13} \mathrm{C}$ NMR $\left(100 \mathrm{MHz}, \mathrm{CDCl}_{3}\right) \quad \delta \quad 161.2,134.3,133.7,120.7,116.4,111.2,101.7,55.9 ;$ HRMS (APCI) $m / z$ calcd for $\mathrm{C}_{8} \mathrm{H}_{8} \mathrm{NO}^{+}[\mathrm{M}+\mathrm{H}]^{+}: 134.0600$ found 134.0602 .

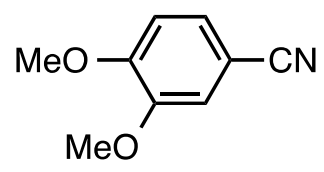

\section{3,4-Dimethoxybenzonitrile (3S) ${ }^{[7]}$}

The reaction was conducted using $\mathrm{NiBr}_{2}(8.7 \mathrm{mg}, 0.040 \mathrm{mmol}, 10 \mathrm{~mol} \%)$, dcypt (38.1 mg, 0.080 mmol, $20 \mathrm{~mol} \%$ ) and Zn powder (15.7 $\mathrm{mg}, 0.24 \mathrm{mmol}, 60 \mathrm{~mol} \%)$. Purification by PTLC (hexane/EtOAc $=3: 1)$ afforded $3 \mathrm{~S}$ as a white solid $\left(52.9 \mathrm{mg}, 81 \%\right.$ yield). ${ }^{1} \mathrm{H}$ NMR $\left(400 \mathrm{MHz}, \mathrm{CDCl}_{3}\right)$ $\delta \quad 7.29(\mathrm{dd}, J=8.4,2.0 \mathrm{~Hz}, 1 \mathrm{H}), 7.08(\mathrm{~d}, J=2.0 \mathrm{~Hz}, 1 \mathrm{H}), 6.91(\mathrm{~d}, J=8.4 \mathrm{~Hz}, 1 \mathrm{H}), 3.94(\mathrm{~s}, 3 \mathrm{H}), 3.91$ 
(s, 3H); ${ }^{13} \mathrm{C}$ NMR $\left(100 \mathrm{MHz}, \mathrm{CDCl}_{3}\right) \quad \delta \quad 152.8,149.1,126.4,119.2,113.9,111.2,103.8,56.1,56.0$; HRMS (APCI) $m / z$ calcd for $\mathrm{C}_{9} \mathrm{H}_{10} \mathrm{NO}_{2}{ }^{+}[\mathrm{M}+\mathrm{H}]^{+}: 164.0706$ found 164.0707.

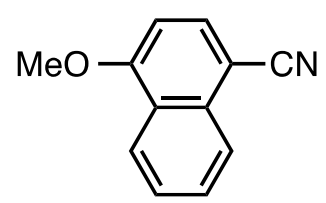

\section{4-Methoxy-1-naphthonitrile (3T) $)^{[13]}$}

Purification by PTLC (hexane/EtOAc $=4: 1$ ) afforded 3 T as a yellow solid ( $44.8 \mathrm{mg}, 61 \%$ yield). ${ }^{1} \mathrm{H}$ NMR $\left(400 \mathrm{MHz}, \mathrm{CDCl}_{3}\right) \quad \delta \quad 8.29(\mathrm{~d}, J=8.4 \mathrm{~Hz}, 1 \mathrm{H}), 8.14(\mathrm{~d}, J=8.4 \mathrm{~Hz}, 1 \mathrm{H}), 7.82(\mathrm{~d}, J=8.4 \mathrm{~Hz}$, $1 \mathrm{H}), 7.67$ (ddd, $J=8.4,7.2,1.6 \mathrm{~Hz}, 1 \mathrm{H}), 7.56(\mathrm{ddd}, J=8.4,7.2,1.2 \mathrm{~Hz}, 1 \mathrm{H}), 6.79(\mathrm{~d}, J=8.0 \mathrm{~Hz}, 1 \mathrm{H})$, 4.04 (s, 3H); ${ }^{13} \mathrm{C}$ NMR (100 MHz, $\left.\mathrm{CDCl}_{3}\right) \quad \delta \quad 159.3,134.0,133.4,128.9,126.6,125.1,124.8,122.7$, 118.4, 103.3, 101.8, 55.9; HRMS (APCI) $\mathrm{m} / z$ calcd for $\mathrm{C}_{12} \mathrm{H}_{10} \mathrm{NO}^{+}[\mathrm{M}+\mathrm{H}]^{+}: 184.0757$ found 184.0758.

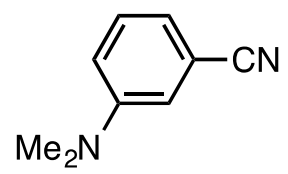

\section{3-(Dimethylamino)benzonitrile (3U) ${ }^{[14]}$}

The reaction was conducted using $\mathrm{NiBr}_{2}(8.7 \mathrm{mg}, 0.040 \mathrm{mmol}, 10 \mathrm{~mol} \%)$, dcypt (38.1 mg, 0.080 mmol, $20 \mathrm{~mol} \%$ ) and $\mathrm{Zn}$ powder (15.7 $\mathrm{mg}, 0.24 \mathrm{mmol}, 60 \mathrm{~mol} \%)$. Purification by PTLC (hexane/EtOAc $=4: 1)$ afforded $\mathbf{3 U}$ as a colorless liquid $\left(44.8 \mathrm{mg}, 77 \%\right.$ yield). ${ }^{1} \mathrm{H}$ NMR $(400 \mathrm{MHz}$, $\left.\mathrm{CDCl}_{3}\right) \quad \delta \quad 7.30-7.24(\mathrm{~m}, 1 \mathrm{H}), 6.94(\mathrm{~d}, J=8.0 \mathrm{~Hz}, 1 \mathrm{H}), 6.90-6.86(\mathrm{~m}, 2 \mathrm{H}), 2.97(\mathrm{~s}, 6 \mathrm{H}) ;{ }^{13} \mathrm{C} \mathrm{NMR}$ $\left(100 \mathrm{MHz}, \mathrm{CDCl}_{3}\right) \quad \delta \quad 150.2,129.7,119.8,119.3,116.2,114.7,112.7,40.1 ; \mathrm{HRMS}$ (APCI) $\mathrm{m} / \mathrm{z}$ calcd for $\mathrm{C}_{9} \mathrm{H}_{11} \mathrm{~N}_{2}^{+}[\mathrm{M}+\mathrm{H}]^{+}: 147.0917$ found 147.0917.

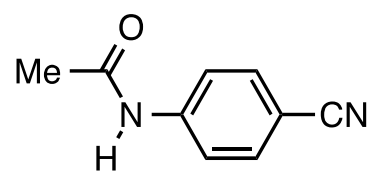

$N$-(4-Cyanophenyl)acetamide $(3 \mathrm{~V})^{[15]}$

Purification by GPC afforded $\mathbf{3 V}$ as a white solid (34.6 mg, 54\% yield). ${ }^{1} \mathrm{H}$ NMR (400 MHz, DMSO- $\left.d_{6}\right) \quad \delta \quad 10.37(\mathrm{~s}, 1 \mathrm{H}), 7.74(\mathrm{bs}, 4 \mathrm{H}), 2.08(\mathrm{~s}, 3 \mathrm{H}) ;{ }^{13} \mathrm{C}$ NMR $\left(100 \mathrm{MHz}, \mathrm{DMSO}-d_{6}\right) \quad \delta \quad 169.2$, 143.5, 133.3, 119.1, 118.9, 104.7, 24.2; HRMS (APCI) $m / z$ calcd for $\mathrm{C}_{9} \mathrm{H}_{7} \mathrm{~N}_{2} \mathrm{O}^{-}[\mathrm{M}-\mathrm{H}]^{-}: 159.0564$ found 159.0552 .

[13] W. Zhou, L. Zhang, N. Jiao, Angew. Chem. Int. Ed. 2009, 48, 7094.

[14] B. K. Lee, M.R. Bicoe, S. L. Buchwald, Tetrahedron Lett. 2009, 50, 3672.

[15] R. Chidambaram, Tetrahedron Lett. 2004, 45, 1441. 


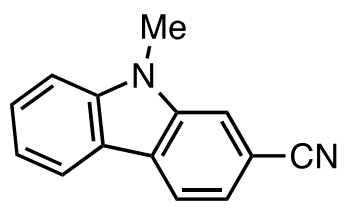

\section{9-Methyl-9H-carbazole-2-carbonitrile (3W)}

Purification by GPC afforded $\mathbf{3 W}$ as a white solid (53.7 mg, 65\% yield). ${ }^{1} \mathrm{H}$ NMR (400 MHz, $\left.\mathrm{CDCl}_{3}\right) \quad \delta \quad 8.10-8.04(\mathrm{~m}, 2 \mathrm{H}), 7.62(\mathrm{~s}, 1 \mathrm{H}), 7.56(\mathrm{ddd}, J=8.4,7.2,1.2 \mathrm{~Hz}, 1 \mathrm{H}), 7.45-7.39(\mathrm{~m}, 2 \mathrm{H})$, $7.29(\mathrm{t}, J=8.0 \mathrm{~Hz}, 1 \mathrm{H}), 3.80(\mathrm{~s}, 3 \mathrm{H}) ;{ }^{13} \mathrm{C} \mathrm{NMR}\left(100 \mathrm{MHz}, \mathrm{CDCl}_{3}\right) \quad \delta \quad 142.0,139.7,127.7,126.1$, 122.0, 121.6, 121.1, 120.8, 120.2, 119.9, 112.5, 108.9, 107.9, 29.2; HRMS (APCI) $\mathrm{m} / \mathrm{z}$ calcd for $\mathrm{C}_{14} \mathrm{H}_{11} \mathrm{~N}_{2}^{+}[\mathrm{M}+\mathrm{H}]^{+}: 207.0917$ found 207.0917.

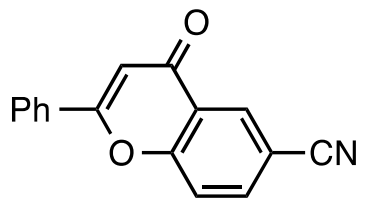

\section{4-Oxo-2-phenyl-4H-chromene-6-carbonitrile (3X)}

The reaction was conducted using $\mathrm{NiBr}_{2}(8.7 \mathrm{mg}, 0.040 \mathrm{mmol}, 10 \mathrm{~mol} \%)$, dcypt (38.1 mg, 0.080 $\mathrm{mmol}, 20 \mathrm{~mol} \%$ ) and $\mathrm{Zn}$ powder (15.7 $\mathrm{mg}, 0.24 \mathrm{mmol}, 60 \mathrm{~mol} \%)$. Purification by GPC afforded $\mathbf{3 X}$ as a white solid (62.0 mg, 63\% yield). ${ }^{1} \mathrm{H}$ NMR $\left(400 \mathrm{MHz}, \mathrm{CDCl}_{3}\right) \quad \delta \quad 8.54(\mathrm{~d}, J=2.0 \mathrm{~Hz}, 1 \mathrm{H}), 7.95-$ $7.90(\mathrm{~m}, 3 \mathrm{H}), 7.69(\mathrm{~d}, J=8.8 \mathrm{~Hz}, 1 \mathrm{H}), 7.62-7.53(\mathrm{~m}, 3 \mathrm{H}), 6.86(\mathrm{~s}, 1 \mathrm{H}) ;{ }^{13} \mathrm{C} \mathrm{NMR}\left(100 \mathrm{MHz}, \mathrm{CDCl}_{3}\right)$ $\delta$ 176.3, 164.0, 157.9, 136.0, 132.2, 131.3, 130.8, 129.2, 126.3, 124.3, 119.7, 117.5, 109.4, 108.0; HRMS (APCI) $m / z$ calcd for $\mathrm{C}_{16} \mathrm{H}_{10} \mathrm{NO}_{2}^{+}[\mathrm{M}+\mathrm{H}]^{+}: 248.0706$ found 248.0705 .

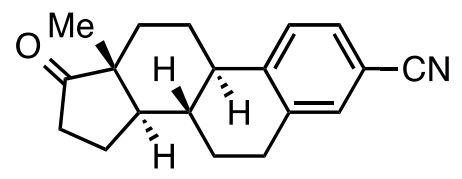

$(8 S, 9 R, 13 R, 14 R)-13-M e t h y l-17-0 x 0-7,8,9,11,12,13,14,15,16,17-d e c a h y d r o-6 H$-cyclopenta $[a]$ phena nthrene-3-carbonitrile (3Y)

Purification by flash silica-gel column chromatography (hexane/EtOAc $=10: 1 \sim 3: 1$ ) afforded $3 Y$ as a white solid (49.1 mg, 44\% yield). ${ }^{1} \mathrm{H}$ NMR (400 MHz, $\left.\mathrm{CDCl}_{3}\right) \quad \delta 7.43(\mathrm{~d}, J=8.0 \mathrm{~Hz}, 1 \mathrm{H})$, 7.40-7.36 (m, 2H), 2.96-2.90 (m, 2H), 2.53 (dd, $J=18.8,8.4 \mathrm{~Hz}, 1 \mathrm{H}), 2.46-2.29(\mathrm{~m}, 2 \mathrm{H}), 2.22-1.96$ $(\mathrm{m}, 4 \mathrm{H}), 1.71-1.41(\mathrm{~m}, 6 \mathrm{H}), 0.92(\mathrm{~s}, 3 \mathrm{H}) ;{ }^{13} \mathrm{C} \mathrm{NMR}\left(100 \mathrm{MHz}, \mathrm{CDCl}_{3}\right) \quad \delta \quad 220.2,145.3,137.9,132.5$, 129.3, 126.2, 119.1, 109.6, 50.4, 47.8, 44.5, 37.6, 35.7, 31.4, 28.9, 25.9, 25.4, 21.5, 13.8; HRMS (APCI) $m / z$ calcd for $\mathrm{C}_{19} \mathrm{H}_{22} \mathrm{NO}^{+}[\mathrm{M}+\mathrm{H}]^{+}: 280.1696$ found 280.1697 . 
<smiles>COC(=O)[C@H](Cc1ccc(C#N)cc1)Pc1ccccc1</smiles>

\section{Methyl (R)-3-(4-cyanophenyl)-2-(1,3-dioxoisoindolin-2-yl)propanoate (3Z)}

The reaction was conducted using $\mathrm{NiBr}_{2}(8.7 \mathrm{mg}, 0.040 \mathrm{mmol}, 10 \mathrm{~mol} \%)$, dcypt (38.1 mg, 0.080 mmol, $20 \mathrm{~mol} \%$ ) and $\mathrm{Zn}$ powder (15.7 $\mathrm{mg}, 0.24 \mathrm{mmol}, 60 \mathrm{~mol} \%)$. Purification by PTLC (hexane/EtOAc $=2: 1$ ) afforded $\mathbf{3 Z}$ as a colorless liquid $\left(91.7 \mathrm{mg}, 69 \%\right.$ yield). ${ }^{1} \mathrm{H}$ NMR $(400 \mathrm{MHz}$, $\left.\mathrm{CDCl}_{3}\right) \delta 7.80(\mathrm{dd}, J=7.4,2.8 \mathrm{~Hz}, 2 \mathrm{H}), 7.73(\mathrm{dd}, J=7.4,2.8 \mathrm{~Hz}, 2 \mathrm{H}), 7.50(\mathrm{~d}, J=8.4 \mathrm{~Hz}, 2 \mathrm{H})$, $7.30(\mathrm{~d}, J=8.4 \mathrm{~Hz}, 2 \mathrm{H}), 5.16(\mathrm{dd}, J=10.8,5.6 \mathrm{~Hz}, 1 \mathrm{H}), 3.79(\mathrm{~s}, 3 \mathrm{H}), 3.70-3.55(\mathrm{~m}, 2 \mathrm{H}) ;{ }^{13} \mathrm{C} \mathrm{NMR}$ $\left(100 \mathrm{MHz}, \mathrm{CDCl}_{3}\right) \quad \delta \quad 168.7,167.3,142.3,134.4,132.4,131.3,129.7,123.6,118.6,110.9,53.1,52.4$, 34.8; HRMS (ESI) $m / z$ calcd for $\mathrm{C}_{19} \mathrm{H}_{14} \mathrm{~N}_{2} \mathrm{O}_{4} \mathrm{Na}^{+}[\mathrm{M}+\mathrm{Na}]^{+}: 357.0846$ found 357.0848.

\section{Ni-Catalyzed Cyanation of Vinyl Carbamate with Aminoacetonitrile 2}

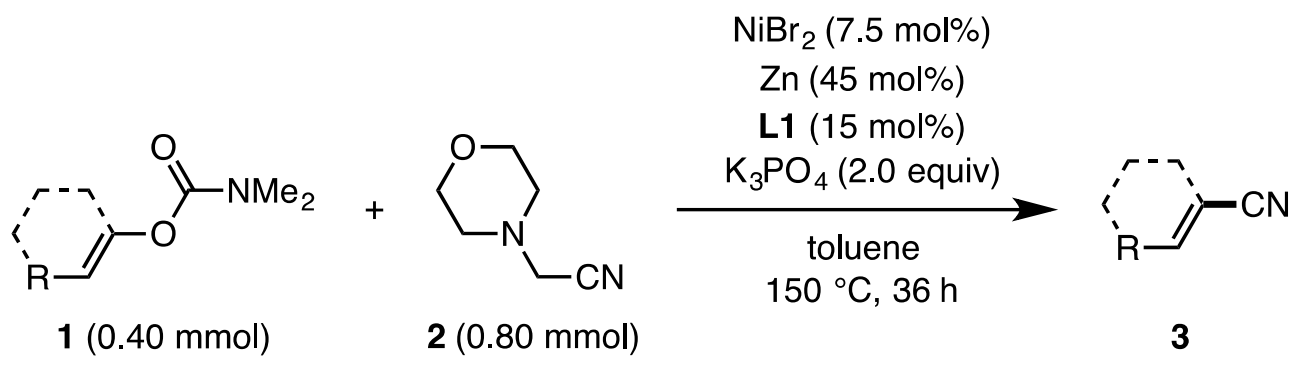

General Procedure: A 20-mL glass vessel equipped with a J. Young® O-ring tap containing a magnetic stirring bar, $\mathrm{K}_{3} \mathrm{PO}_{4}(169.8 \mathrm{mg}, 0.80 \mathrm{mmol}, 2.0$ equiv) and $\mathrm{Zn}$ powder (11.8 mg, $0.18 \mathrm{mmol}$, $45 \mathrm{~mol} \%$ ) were dried with a heatgun for $3 \mathrm{~min}$ in vacuo and filled with $\mathrm{N}_{2}$ after cooling to room temperature. To this vessel were added $\mathrm{NiBr}_{2}(6.6 \mathrm{mg}, 0.030 \mathrm{mmol}, 7.5 \mathrm{~mol} \%)$, vinyl carbamates 1 (0.40 mmol, 1.0 equiv), 2-morpholinoacetonitrile 2 (100.9 mg, $0.80 \mathrm{mmol}, 2.0$ equiv) and 2,3-bis(dicyclohexylphosphino)thiophene (L1: $28.6 \mathrm{mg}, 0.060 \mathrm{mmol}, 15 \mathrm{~mol} \%$ ). Then, dry toluene $(1.6 \mathrm{~mL})$ was added under a stream of $\mathrm{N}_{2}$. The vessel was sealed with an O-ring tap and then heated at $150{ }^{\circ} \mathrm{C}$ for $18 \mathrm{~h}$ in an 8 -well reaction block with stirring. After cooling the reaction mixture to room temperature, the mixture was passed through a silica gel pad with EtOAc as the eluent. The filtrate was concentrated and the residue was subjected to PTLC, flash silica-gel column chromatography or GPC to afford vinyl cyanides 3 .

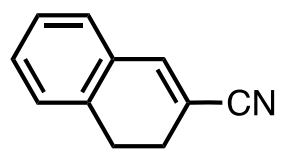

\section{9-Methyl-9H-carbazole-2-carbonitrile (3AA)}

Purification by PTLC (hexane/EtOAc $=6: 1$ ) afforded 3AA as a yellow solid $(42.2 \mathrm{mg}, 68 \%$ yield). ${ }^{1} \mathrm{H}$ NMR (400 MHz, $\left.\mathrm{CDCl}_{3}\right) \quad \delta 7.31-7.20(\mathrm{~m}, 2 \mathrm{H}), 7.20-7.11(\mathrm{~m}, 3 \mathrm{H}), 2.90(\mathrm{t}, J=8.8 \mathrm{~Hz}$, 
2H), $2.54(\operatorname{td}, J=8.0,1.6 \mathrm{~Hz}, 2 \mathrm{H}) ;{ }^{13} \mathrm{C} \mathrm{NMR}\left(100 \mathrm{MHz}, \mathrm{CDCl}_{3}\right) \quad \delta \quad 141.6,135.4,131.1,130.2,127.9$, 127.1 (one peak is overlapping), 119.6, 109.6, 26.6, 24.6; HRMS (APCI) $m / z$ calcd for $\mathrm{C}_{11} \mathrm{H}_{10} \mathrm{~N}^{+}[\mathrm{M}+$ $\mathrm{H}]^{+}: 156.0808$ found 156.0809 .

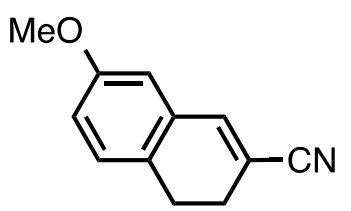

\section{9-Methyl-9H-carbazole-2-carbonitrile (3AB)}

Purification by PTLC (hexane/EtOAc $=4: 1$ ) afforded $\mathbf{3 A B}$ as a white solid ( $49.5 \mathrm{mg}, 67 \%$ yield). ${ }^{1} \mathrm{H}$ NMR $\left(400 \mathrm{MHz}, \mathrm{CDCl}_{3}\right) \quad \delta 7.12(\mathrm{~s}, 1 \mathrm{H}), 7.07(\mathrm{~d}, J=8.4 \mathrm{~Hz}, 1 \mathrm{H}), 6.82(\mathrm{dd}, J=8.4,2.8 \mathrm{~Hz}, 1 \mathrm{H})$, $6.69(\mathrm{~d}, J=2.8 \mathrm{~Hz}, 1 \mathrm{H}), 3.79(\mathrm{~s}, 3 \mathrm{H}), 2.82(\mathrm{t}, J=8.8 \mathrm{~Hz}, 2 \mathrm{H}), 2.51(\mathrm{td}, J=8.8 \mathrm{~Hz}, 1.6 \mathrm{~Hz}, 2 \mathrm{H}) ;{ }^{13} \mathrm{C}$ $\operatorname{NMR}\left(100 \mathrm{MHz}, \mathrm{CDCl}_{3}\right) \quad \delta \quad 158.5,141.6,131.9,128.8,127.4,119.6,115.5,113.3,110.2,55.4,25.8$, 25.0; HRMS (APCI) $m / z$ calcd for $\mathrm{C}_{12} \mathrm{H}_{12} \mathrm{NO}^{+}[\mathrm{M}+\mathrm{H}]^{+}$: 186.0913 found 186.0915.

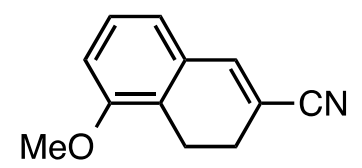

\section{5-Methoxy-3,4-dihydronaphthalene-2-carbonitrile (3AC)}

Purification by PTLC (hexane/EtOAc $=4: 1)$ afforded $\mathbf{3 A C}$ as a colorless liquid $(47.1 \mathrm{mg}, 64 \%$ yield). ${ }^{1} \mathrm{H} \mathrm{NMR}\left(400 \mathrm{MHz}, \mathrm{CDCl}_{3}\right) \quad \delta \quad 7.19(\mathrm{t}, J=8.0 \mathrm{~Hz}, 1 \mathrm{H}), 7.14(\mathrm{t}, J=1.6 \mathrm{~Hz}, 1 \mathrm{H}), 6.89(\mathrm{~d}, J=$ $8.0 \mathrm{~Hz}, 1 \mathrm{H}), 6.77(\mathrm{~d}, J=8.0 \mathrm{~Hz}, 1 \mathrm{H}), 3.84(\mathrm{~s}, 3 \mathrm{H}), 2.88(\mathrm{t}, J=8.4 \mathrm{~Hz}, 2 \mathrm{H}), 2.53-2.46(\mathrm{~m}, 2 \mathrm{H}) ;{ }^{13} \mathrm{C}$ NMR $\left(100 \mathrm{MHz}, \mathrm{CDCl}_{3}\right) \quad \delta \quad 156.1,141.6,131.9,127.3,123.2,120.5,119.7,112.6,109.7,55.5,24.1$, 19.0; HRMS (APCI) $m / z$ calcd for $\mathrm{C}_{12} \mathrm{H}_{12} \mathrm{NO}^{+}[\mathrm{M}+\mathrm{H}]^{+}: 186.0913$ found 186.0915 .

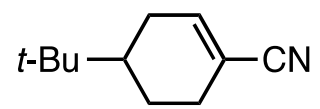

\section{4-(tert-Butyl)cyclohex-1-ene-1-carbonitrile (3AD) $)^{[16]}$}

Purification by flash silica-gel column chromatography (hexane/EtOAc $=50: 1 \sim 5: 1$ ) afforded 3AD as a white solid (49.6 mg, 76\% yield). ${ }^{1} \mathrm{H}$ NMR (400 $\left.\mathrm{MHz}, \mathrm{CDCl}_{3}\right) \quad \delta \quad 6.66-6.63(\mathrm{~m}, 1 \mathrm{H})$, 2.37-2.16 (m, 3H), 1.97-1.86 (m, 2H), 1.33-1.12 (m, 2H), $0.88(\mathrm{~s}, 9 \mathrm{H}) ;{ }^{13} \mathrm{C} \mathrm{NMR}\left(100 \mathrm{MHz}, \mathrm{CDCl}_{3}\right)$ $\delta \quad 145.6,119.7,112.1,42.6,32.1,28.0,27.6,26.9,23.0$; HRMS (APCI) $m / z$ calcd for $\mathrm{C}_{11} \mathrm{H}_{18} \mathrm{~N}^{+}[\mathrm{M}+$ $\mathrm{H}]^{+}: 164.1434$ found 164.1435 .

\section{Sequential Cross-coupling for the Synthesis of 5CB}

[16] M. Ochiai, K. Sumi, Y. Takaoka, M. Kunishima, Y. Nagao, M. Shiro, E. Fujita, Tetrahedron 1988, 44, 4095 . 


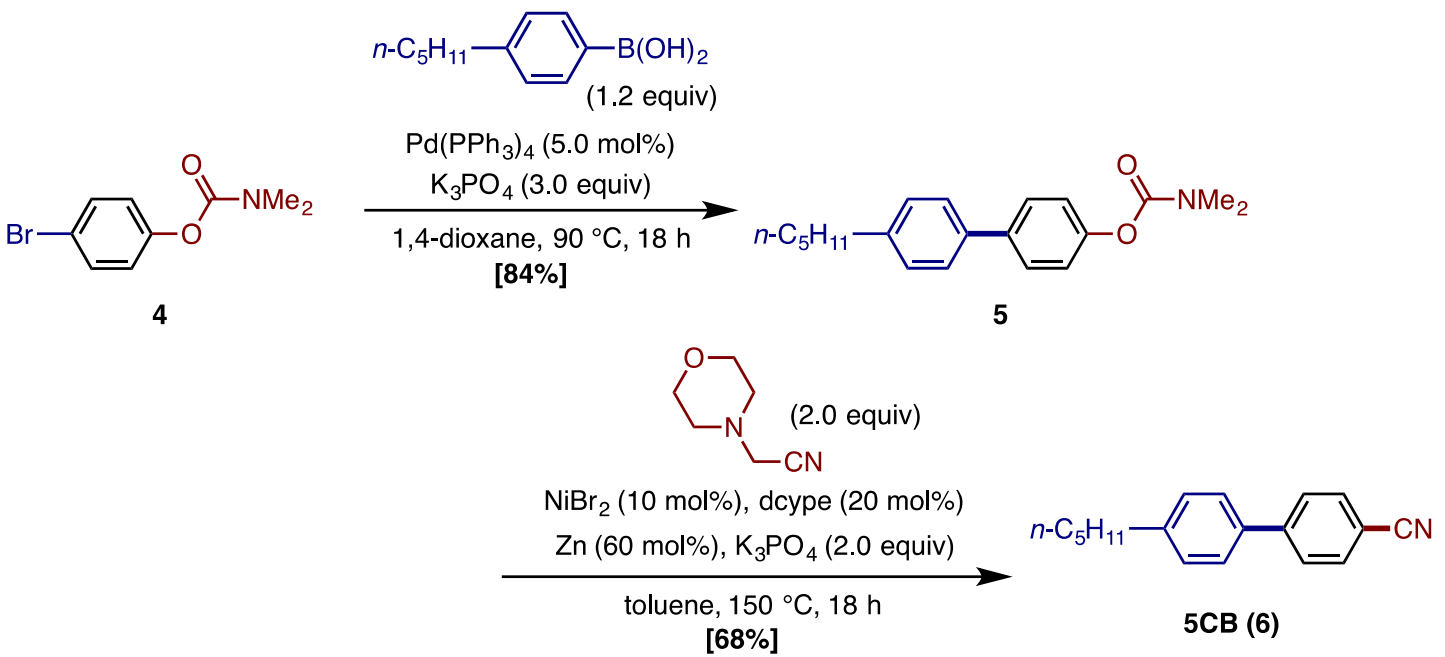

Note: $\mathbf{4}^{[17]}$ was synthesized according to procedures reported in the literature.

To a 20-mL Schlenk flask, containing a magnetic stirring bar, was added 4-bromophenyl dimethylcarbamate 4 (122.0 mg, $0.50 \mathrm{mmol}, 1.0$ equiv), (4-pentylphenyl)boronic acid (115.2 mg, 0.60 mmol, 1.2 equiv), $\mathrm{Pd}\left(\mathrm{PPh}_{3}\right)_{4}(28.9 \mathrm{mg}, 0.025 \mathrm{mmol}, 5.0 \mathrm{~mol} \%), \mathrm{K}_{3} \mathrm{PO}_{4}(318.4 \mathrm{mg}, 1.5 \mathrm{mmol}, 3.0$ equiv) and a dry 1,4-dioxane $(4.0 \mathrm{~mL})$ under a stream of $\mathrm{N}_{2}$. The flask was heated at $90{ }^{\circ} \mathrm{C}$ for $18 \mathrm{~h}$. After cooling the reaction mixture to room temperature, the mixture was passed through a short silica gel pad with EtOAc. The filtrate was concentrated in vacuo, and the residue was purified by flash silica-gel column chromatography (hexane/EtOAc $=20: 1 \sim 5: 1$ ) to afford 4'-pentyl-[1,1'-biphenyl]-4-yl dimethylcarbamate 5 as a white solid (131.2 $\mathrm{mg}, 84 \%$ yield). ${ }^{1} \mathrm{H}$ NMR $\left(400 \mathrm{MHz}, \mathrm{CDCl}_{3}\right): \delta 7.55(\mathrm{~d}, J=8.8 \mathrm{~Hz}, 2 \mathrm{H}), 7.47(\mathrm{~d}, J=8.4 \mathrm{~Hz}, 2 \mathrm{H}), 7.24(\mathrm{~d}, J=8.4 \mathrm{~Hz}, 2 \mathrm{H}), 7.16$ $(\mathrm{d}, J=8.8 \mathrm{~Hz}, 2 \mathrm{H}), 3.12(\mathrm{~s}, 3 \mathrm{H}), 3.03(\mathrm{~s}, 3 \mathrm{H}), 2.63(\mathrm{t}, J=7.6 \mathrm{~Hz}, 2 \mathrm{H}), 1.65(\mathrm{q}, J=7.6 \mathrm{~Hz}, 2 \mathrm{H}), 1.40$ $1.30(\mathrm{~m}, 4 \mathrm{H}), 0.90(\mathrm{t}, J=7.2 \mathrm{~Hz}, 3 \mathrm{H}) ;{ }^{13} \mathrm{C} \mathrm{NMR}\left(100 \mathrm{MHz}, \mathrm{CDCl}_{3}\right): \delta 154.9,150.7,142.0,138.2$, 137.9, 128.8, 127.8, 126.9, 121.9, 36.7, 36.4, 35.5, 31.5, 31.2, 22.5, 14.0; HRMS (ESI) $\mathrm{m} / \mathrm{z}$ calcd for $\mathrm{C}_{20} \mathrm{H}_{25} \mathrm{NO}_{2} \mathrm{Na}^{+}[\mathrm{M}+\mathrm{Na}]^{+}: 334.1778$ found 334.1774 .

A $20-\mathrm{mL}$ glass vessel equipped with a J. Young® O-ring tap containing a magnetic stirring bar, $\mathrm{K}_{3} \mathrm{PO}_{4}$ (127.4 mg, $0.60 \mathrm{mmol}, 2.0$ equiv) and $\mathrm{Zn}$ powder (11.8 mg, $0.18 \mathrm{mmol}, 60 \mathrm{~mol} \%)$ were dried with a heat gun for $3 \mathrm{~min}$ in vacuo and filled with $\mathrm{N}_{2}$ after cooling to room temperature. To this vessel were added $\mathrm{NiBr}_{2}$ (6.6 mg, $\left.0.030 \mathrm{mmol}, 10 \mathrm{~mol} \%\right)$, carbamate 5 (93.4 mg, $0.30 \mathrm{mmol}, 1.0$ equiv), 2-morpholinoacetonitrile $2(75.7 \mathrm{mg}, 0.60 \mathrm{mmol}, 2.0$ equiv). After the vessel was introduced into an argon-atmosphere glovebox, to the reaction vessel was added 1,2-bis(dicyclohexylphosphino)ethane (dcype: $25.4 \mathrm{mg}, 0.060 \mathrm{mmol}, 20 \mathrm{~mol} \%$ ) and dry toluene $(1.2 \mathrm{~mL}$ ), and then the vessel was taken out of the glovebox. The vessel was sealed with an O-ring tap and then heated at $150{ }^{\circ} \mathrm{C}$ for $18 \mathrm{~h}$ in an 8 -well reaction block with stirring. After cooling the reaction mixture to room temperature, the mixture was passed through a silica gel pad with EtOAc as the eluent. The filtrate was concentrated

[17] A. John, K. M. Nicholas, J. Org. Chem. 2012, 77, 5600. 
and the residue was subjected to PTLC (hexane/EtOAc $=4: 1$ ), to afford 6 as a yellow liquid $\left(5 \mathrm{CB}^{[18]}\right.$ : $50.6 \mathrm{mg}, 68 \%) .{ }^{1} \mathrm{H} \mathrm{NMR}\left(400 \mathrm{MHz}, \mathrm{CDCl}_{3}\right): \delta 7.72-7.63(\mathrm{~m}, 4 \mathrm{H}), 7.50(\mathrm{~d}, J=8.8 \mathrm{~Hz}, 2 \mathrm{H}), 7.29(\mathrm{~d}, J$ $=8.4 \mathrm{~Hz}, 2 \mathrm{H}), 2.65(\mathrm{t}, J=8.0 \mathrm{~Hz}, 2 \mathrm{H}), 1.70-1.60(\mathrm{~m}, 2 \mathrm{H}), 1.40-1.28(\mathrm{~m}, 4 \mathrm{H}), 0.90(\mathrm{t}, J=7.2 \mathrm{~Hz}$, $3 \mathrm{H}) ;{ }^{13} \mathrm{C}$ NMR (100 MHz, $\left.\mathrm{CDCl}_{3}\right): \delta 145.6,143.8,136.4,132.5,129.1,127.4,127.0,119.0,110.4$, 35.5, 31.4, 31.0, 22.5, 14.0; HRMS (APCI) $\mathrm{m} / z$ calcd for $\mathrm{C}_{18} \mathrm{H}_{20} \mathrm{~N}^{+}[\mathrm{M}+\mathrm{H}]^{+}: 250.1590$ found 250.1590 .

\section{Ligand Screening}

Note: Reaction procedure is same as general procedure of Ni-catalyzed cyanation of vinyl carbamate with aminoacetonitriles in chapter 5.
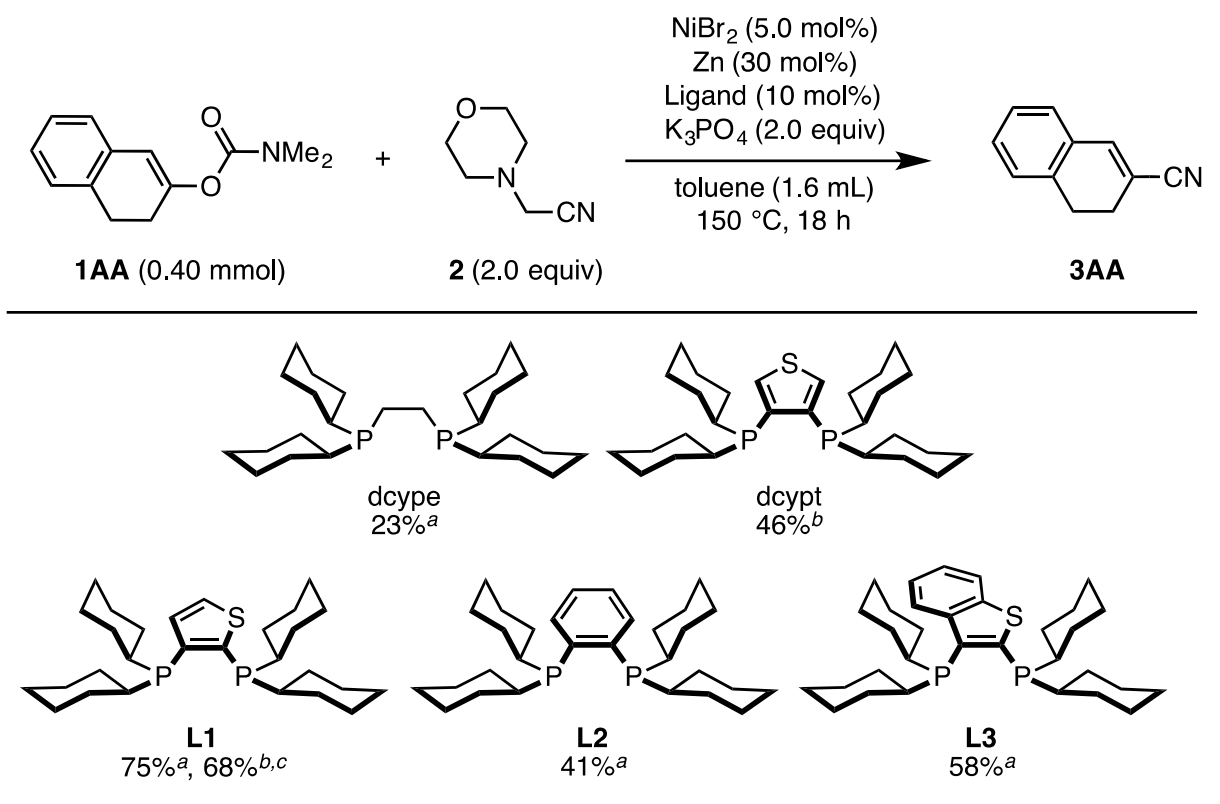

a NMR yield

$\mathrm{b}$ isolated yield

c the reaction was conducted with $\mathrm{NiBr}_{2}$ (7.5 mol\%), L3 (15 mol\%), Zn (0.45 equiv) for $36 \mathrm{~h}$.

[18] S. Ushijima, K. Moriyama, H. Togo, Tetrahedron 2011, 67, 958. 